\title{
Whole-genome association analyses of sleep-disordered breathing phenotypes in the NHLBI TOPMed program
}

Brian E. Cade ${ }^{1,2,3^{*}}$ (D), Jiwon Lee ${ }^{1}$, Tamar Sofer ${ }^{1,2}$, Heming Wang ${ }^{1,2,3}$, Man Zhang ${ }^{4}$, Han Chen ${ }^{5,6}$, Sina A. Gharib ${ }^{7}$, Daniel J. Gottlieb ${ }^{1,2,8}$, Xiuqing Guo ${ }^{9}$, Jacqueline M. Lane ${ }^{1,2,3,10}$, Jingjing Liang ${ }^{11}$, Xihong Lin ${ }^{12}$, Hao Mei ${ }^{13}$, Sanjay R. Patel ${ }^{14}$, Shaun M. Purcell ${ }^{1,2,3}$, Richa Saxena ${ }^{1,2,3,10}$, Neomi A. Shah ${ }^{15}$, Daniel S. Evans ${ }^{16}$, Craig L. Hanis ${ }^{5}$, David R. Hillman ${ }^{17}$, Sutapa Mukherjee ${ }^{18,19}$, Lyle J. Palmer ${ }^{20}$, Katie L. Stone ${ }^{16}$, Gregory J. Tranah ${ }^{16}$, NHLBI Trans-Omics for Precision Medicine (TOPMed) Consortium, Gonçalo R. Abecasis ${ }^{21}$, Eric A. Boerwinkle ${ }^{5,22}$, Adolfo Correa ${ }^{23,24}$, L. Adrienne Cupples ${ }^{25,26}$, Robert C. Kaplan ${ }^{27}$, Deborah A. Nickerson ${ }^{28,29}$, Kari E. North ${ }^{30}$, Bruce M. Psaty ${ }^{31,32}$, Jerome I. Rotter ${ }^{9}$, Stephen S. Rich ${ }^{33}$, Russell P. Tracy ${ }^{34}$, Ramachandran S. Vasan ${ }^{26,35,36}$, James G. Wilson ${ }^{37}$, Xiaofeng Zhu ${ }^{11}$, Susan Redline ${ }^{1,2,38}$ and TOPMed Sleep Working Group

\begin{abstract}
Background: Sleep-disordered breathing is a common disorder associated with significant morbidity. The genetic architecture of sleep-disordered breathing remains poorly understood. Through the NHLBI Trans-Omics for Precision Medicine (TOPMed) program, we performed the first whole-genome sequence analysis of sleep-disordered breathing.

Methods: The study sample was comprised of 7988 individuals of diverse ancestry. Common-variant and pathway analyses included an additional 13,257 individuals. We examined five complementary traits describing different aspects of sleep-disordered breathing: the apnea-hypopnea index, average oxyhemoglobin desaturation per event, average and minimum oxyhemoglobin saturation across the sleep episode, and the percentage of sleep with oxyhemoglobin saturation $<90 \%$. We adjusted for age, sex, BMI, study, and family structure using MMSKAT and EMMAX mixed linear model approaches. Additional bioinformatics analyses were performed with MetaXcan, GIGSEA, and ReMap.
\end{abstract}

\footnotetext{
* Correspondence: bcade@bwh.harvard.edu

Full lists of consortium and working group authors are provided in Additional file 1: Tables S1 and S2.

'Division of Sleep and Circadian Disorders, Brigham and Women's Hospital,

Harvard Medical School, 221 Longwood Avenue, Boston, MA 02115, USA

2Division of Sleep Medicine, Harvard Medical School, Boston, MA 02115, USA

Full list of author information is available at the end of the article
}

C C The Author(s). 2021 Open Access This article is licensed under a Creative Commons Attribution 4.0 International License, which permits use, sharing, adaptation, distribution and reproduction in any medium or format, as long as you give appropriate credit to the original author(s) and the source, provide a link to the Creative Commons licence, and indicate if changes were made. The images or other third party material in this article are included in the article's Creative Commons licence, unless indicated otherwise in a credit line to the material. If material is not included in the article's Creative Commons licence and your intended use is not permitted by statutory regulation or exceeds the permitted use, you will need to obtain permission directly from the copyright holder. To view a copy of this licence, visit http://creativecommons.org/licenses/by/4.0/. The Creative Commons Public Domain Dedication waiver (http://creativecommons.org/publicdomain/zero/1.0/) applies to the data made available in this article, unless otherwise stated in a credit line to the data. 
Results: We identified a multi-ethnic set-based rare-variant association $\left(p=3.48 \times 10^{-8}\right)$ on chromosome $X$ with ARMCX3. Additional rare-variant associations include ARMCX3-AS1, MRPS33, and C16orf90. Novel common-variant loci were identified in the NRG1 and SLC45A2 regions, and previously associated loci in the IL18RAP and ATP2B4 regions were associated with novel phenotypes. Transcription factor binding site enrichment identified associations with genes implicated with respiratory and craniofacial traits. Additional analyses identified significantly associated pathways.

Conclusions: We have identified the first gene-based rare-variant associations with objectively measured sleepdisordered breathing traits. Our results increase the understanding of the genetic architecture of sleep-disordered breathing and highlight associations in genes that modulate lung development, inflammation, respiratory rhythmogenesis, and HIF1A-mediated hypoxic response.

Keywords: Sleep-disordered breathing, Sleep apnea, Whole-genome sequencing, WGS, Genome-wide association study, GWAS

\section{Background}

Sleep-disordered breathing (SDB) is a prevalent disorder associated with increased sleepiness, mortality, and morbidity from a wide range of cardiometabolic and other diseases $[1,2]$. The most common type of SDB is obstructive sleep apnea (OSA), characterized by repeated airway collapse leading to intermittent hypoxemia and sleep disruption, that is increased in prevalence with older age and male sex [2]. An estimated 936 million adults aged 30-69 have mild to severe OSA worldwide [3]. The disease is heritable and appears to be multifactorial, reflecting variable contributions of abnormalities in ventilatory control, craniofacial anatomy, and adiposity [2, 4-7]. Sleep-related hypoxemia can also be due to central sleep apnea, a less common disorder, due to a lack of respiratory drive [8]. OSA is typically measured clinically using the apnea-hypopnea index, which counts the number of total (apnea) and partial (hypopnea) breathing cessations per hour of sleep. Due to an incomplete understanding of its molecular basis, the standard OSA treatment of continuous positive airway pressure (CPAP) only addresses the downstream manifestations of airway collapse through nightly use of pressurized air to the nasopharynx, a therapy that often is poorly tolerated. Therefore, there is a critical need to identify molecular pathways that could provide specific therapeutic targets. The need for overnight studies to phenotype SDB traits has limited the available sample size for genetic analyses, and only several common-frequency genome-wide analysis studies have been reported [911]. Increased statistical power may increase the genetic resolution of regions that may not be adequately tagged by current genotyping arrays due to population differences and/or reduced linkage disequilibrium with biologically relevant regions.

The Trans-Omics for Precision Medicine (TOPMed) program is an NIH National Heart, Lung, and Blood
Institute program designed to improve the understanding of the biological processes that contribute to heart, lung, blood, and sleep disorders [12]. TOPMed has generated whole-genome sequencing (WGS) data on over 100,000 individuals from multiple cohorts at $>30 \times$ depth, including seven studies with objective assessment of SDB. A variant imputation server using TOPMed data also allows for high-quality imputation of nonsequenced genotype chip data [13]. A complementary initiative sponsored by the Centers for Common Disease Genomics (CCDG) of the NIH National Human Genome Research Institute has generated sequencing data from additional individuals in two TOPMed cohorts. These initiatives provide the ability to examine the genetics of SDB at unprecedented detail in African-Americans (AA), Asian-Americans (AsA), European-Americans/Australians (EA), and Hispanic/Latino-Americans (HA).

In this first genome-wide sequencing analysis of SDB, we examine the apnea-hypopnea index (AHI), the standard clinic metric of SDB, and four complementary measurements of overnight hypoxemia: average and minimum oxyhemoglobin saturation $\left(\mathrm{SpO}_{2}\right)$ during sleep and the percent of the sleep recording with $\mathrm{SpO}_{2}<90 \%$ (Per90), and the average desaturation per hypopnea event. These indices were chosen because of clinical relevance, high heritability, or prior significant GWAS findings $[9,11,14]$. We examined 7988 individuals with objectively measured SDB and WGS data in conjunction with data from 13,257 individuals with imputed genotype data.

\section{Methods}

Each study had a protocol approved by its respective Institutional Review Board and participants provided informed consent. A study overview is provided in Additional file 2: Figure S1. There were two classes of data: "WGS studies" had WGS performed by the TOPMed 
program and, in some cases, in additional participants by the CCDG program (referred to as "WGS" studies); "Imputed studies" had array-based genotyping later imputed using the TOPMed imputation server (as described below). Some studies with WGS contributed imputed study data from additional array-based genotyped individuals. Ten studies were analyzed (Tables 1 and 2).

\section{WGS studies}

The Atherosclerosis Risk in Communities Study (ARIC), the Cardiovascular Health Study (CHS), and the Framingham Heart Study Offspring Cohort (FHS) included individuals who participated in the Sleep Heart Health Study (SHHS), who underwent polysomnography (PSG) between 1995 and 1998 using the Compumedics PS-2 system [15-18]. These samples included 1028 EAs from ARIC, 151 AAs and 557 EAs from CHS, and 478 EAs from FHS.

The Multi-Ethnic Study of Atherosclerosis (MESA) is investigating the risk factors for clinical cardiovascular disease [19]. PSG was obtained between 2010 and 2013 using the Compumedics Somte system [20]. This analysis includes data from $698 \mathrm{EAs}, 486$ AAs, $456 \mathrm{HAs}$, and 229 AsAs.

The Cleveland Family Study (CFS) was designed to investigate the familial basis of SDB, with four visits occurring from 1990 to 2006 [21]. Sleep was assessed either in a clinical research center using full PSG (Compumedics E series) (visit 4) or in the latest available prior examination using an in-home sleep apnea testing device (Edentrace). Data were analyzed from 505 AAs and 485 EAs (339 AAs and 234 EAs with full PSG data).
The Hispanic Community Health Study/Study of Latinos $(\mathrm{HCHS} / \mathrm{SOL})$ is studying multiple health conditions in HAs [22, 23]. Home sleep apnea testing was performed during the baseline examination (2008-2011) using the ARES Unicorder 5.2, a validated device including a forehead-based reflectance oximeter, a nasal pressure cannula and pressure transducer, an accelerometer, and a microphone [24]. Two thousand three hundred thirty-nine individuals provided data.

The Jackson Heart Study (JHS) is investigating cardiovascular disease in AAs [25]. An in-home sleep study was performed from 2012 to 2016 using a validated type 3 sleep apnea testing device (Embla Embletta Gold) [26, 27]. Five hundred seventy-five individuals contributed data.

\section{Imputed genotype studies}

The Osteoporotic Fractures in Men Study (MrOS) is a multi-center cohort study initially designed to examine the risk factors for osteoporosis, fractures, and prostate cancer in older males $[28,29]$. An ancillary study (MrOS Sleep; 2003-2005) focused on outcomes of sleep disturbances used PSG and nearly identical procedures as in MESA (Compumedics Safiro system) [30]. Two thousand one hundred eighty-one EA individuals were included, with genotyping performed using the Illumina Human Omni 1 Quad v1-0 H array.

The Starr County Health Studies (Starr) investigates the risk factors for diabetes in Mexican-Americans [31, 32]. An in-home sleep apnea study occurred between 2010 and 2014 using a validated instrument that records finger pulse oximetry, actigraphy, body position, and peripheral arterial tonometry (Itamar-Medical

Table 1 Sample description for WGS cohorts

\begin{tabular}{|c|c|c|c|c|c|c|c|c|c|c|c|}
\hline Population & Cohort & $\mathbf{N}$ & Age & $\begin{array}{l}\text { Percent } \\
\text { female }\end{array}$ & BMI & $\begin{array}{l}\text { Apnea- } \\
\text { hypopnea } \\
\text { index } 3 \%\end{array}$ & $\begin{array}{l}\text { AHI (percent } \\
<5,5-15, \geq 15)\end{array}$ & $\begin{array}{l}\text { Average } \\
\text { desaturation }\end{array}$ & $\begin{array}{l}\text { Average } \\
\mathrm{SpO}_{2}\end{array}$ & $\begin{array}{l}\text { Minimum } \\
\mathrm{SpO}_{2}\end{array}$ & $\begin{array}{l}\text { Percent } \\
\text { sleep under } \\
90 \% \mathrm{SpO}_{2}\end{array}$ \\
\hline \multirow{4}{*}{$\begin{array}{l}\text { African- } \\
\text { American }\end{array}$} & $\mathrm{CFS}^{*}$ & 505 & 38.65 (18.96) & 56.4 & $32.44(9.48)$ & $6.85(22.48)$ & $43.4,20.6,36.0$ & 3.62 (1.99) & $94.49(3.91)$ & $84.76(9.83)$ & 4.79 (13.15) \\
\hline & $\mathrm{CHS}$ & 151 & $75.39(4.35)$ & 60.3 & $29.02(5.08)$ & $9.60(16.96)$ & $28.5,36.4,35.1$ & $2.70(1.74)$ & $94.82(2.19)$ & $85.74(5.35)$ & 3.39 (9.63) \\
\hline & JHS & 575 & $63.47(10.94)$ & 64.9 & $31.8(6.88)$ & 10.69 (14.42) & $24.7,39.5,35.8$ & 3.54 (1.72) & 94.77 (2.02) & $84.30(6.57)$ & $2.97(8.91)$ \\
\hline & MESA & 486 & $68.81(9.07)$ & 53.7 & $30.23(5.68)$ & $12.67(20.56)$ & $22.4,32.9,44.7$ & $3.42(2.10)$ & 94.46 (1.99) & $83.32(7.98)$ & 3.89 (9.49) \\
\hline $\begin{array}{l}\text { East Asian- } \\
\text { American }\end{array}$ & MESA & 229 & $67.89(9.11)$ & 49.8 & $24.28(3.30)$ & $14.96(24.28)$ & $21.8,28.4,49.8$ & $3.72(1.79)$ & $94.92(1.22)$ & $83.23(7.58)$ & $2.25(4.46)$ \\
\hline \multirow{5}{*}{$\begin{array}{l}\text { European- } \\
\text { American }\end{array}$} & ARIC & 1028 & $62.28(5.67)$ & 53.1 & $28.72(5.06)$ & $8.64(15.62)$ & $34.6,32.4,33.0$ & $2.35(1.29)$ & $94.57(1.84)$ & 85.95 (5.93) & $2.92(9.24)$ \\
\hline & $\mathrm{CFS}^{*}$ & 485 & 43.23 (19.49) & 50.5 & $30.81(8.83)$ & 7.09 (21.90) & $44.7,19.4,35.9$ & $3.29(1.86)$ & 93.67 (3.59) & $85.55(9.33)$ & 4.66 (11.87) \\
\hline & $\mathrm{CHS}$ & 557 & $77.90(4.34)$ & 54.2 & $27.25(4.44)$ & $11.42(15.54)$ & $23.2,38.1,38.8$ & $2.58(1.34)$ & $94.00(2.00)$ & 84.99 (5.67) & $4.77(12.28)$ \\
\hline & $\mathrm{FHS}^{*}$ & 478 & $60.09(8.54)$ & 49.8 & $28.40(5.06)$ & $8.10(14.28)$ & $35.1,35.1,29.7$ & $2.35(1.27)$ & $94.68(2.04)$ & $85.78(6.25)$ & $2.96(9.18)$ \\
\hline & MESA & 698 & $68.53(9.06)$ & 53.2 & $27.91(5.10)$ & $12.18(20.45)$ & $21.6,35.0,43.4$ & $3.11(1.44)$ & $93.96(1.75)$ & $83.49(7.50)$ & $4.27(10.82)$ \\
\hline \multirow{2}{*}{$\begin{array}{l}\text { Hispanic/Latino- } \\
\text { American }\end{array}$} & $\mathrm{HCHS} / \mathrm{SOL}$ & 2339 & $46.27(13.86)$ & 60.5 & $30.23(6.44)$ & $2.03(6.30)$ & $68.9,19.5,11.6$ & N/A & $96.42(0.99)$ & $87.04(5.92)$ & $0.88(3.63)$ \\
\hline & MESA & 456 & $68.49(9.27)$ & 53.3 & $30.08(5.46)$ & $16.31(22.53)$ & $17.1,28.3,54.6$ & $3.62(2.12)$ & $94.33(1.60)$ & $81.59(9.32)$ & $3.80(7.64)$ \\
\hline
\end{tabular}

Seven studies contributed 7988 individuals with WGS in TOPMed Freeze 6a and objectively measured phenotypes (1717 African-Americans, 229 Asian-Americans, 3246 European-Americans, 2796 Hispanic/Latino-Americans). The overall sample had a mean age of 57.7 and was $56.1 \%$ female. Values are displayed as mean (SD), except for the skewed apnea-hypopnea index, which is displayed as median (IQR). Sample size N reflects individuals with non-missing AHI and covariate values. *Family cohort 
Table 2 Sample description for imputed genotype chip cohorts

\begin{tabular}{|c|c|c|c|c|c|c|c|c|c|c|c|}
\hline Population & Cohort & $\mathrm{N}$ & Age & $\begin{array}{l}\text { Percent } \\
\text { female }\end{array}$ & BMI & $\begin{array}{l}\text { Apnea- } \\
\text { hypopnea } \\
\text { index } 3 \%\end{array}$ & $\begin{array}{l}\text { AHI (percent } \\
<5,5-15, \geq 15 \text { ) }\end{array}$ & $\begin{array}{l}\text { Average } \\
\text { desaturation }\end{array}$ & $\begin{array}{l}\text { Average } \\
\mathrm{SpO}_{2}\end{array}$ & $\begin{array}{l}\text { Minimum } \\
\mathrm{SpO}_{2}\end{array}$ & $\begin{array}{l}\text { Percent sleep } \\
\text { under } 90 \% \\
\mathrm{SpO}_{2}\end{array}$ \\
\hline African-American & $\mathrm{CFS}^{*}$ & 225 & $35.46(20.32)$ & 56.4 & $29.97(10.09)$ & $3.99(10.55)$ & $55.1,23.1,21.8$ & $2.90(1.09)$ & $94.65(4.01)$ & $88.17(9.60)$ & $5.20(16.01)$ \\
\hline \multirow{6}{*}{$\begin{array}{l}\text { European- } \\
\text { American, } \\
\text { Australian }\end{array}$} & ARIC & 631 & $62.74(5.72)$ & 49.4 & $29.15(5.23)$ & $9.15(15.02)$ & $29.3,37.9,32.8$ & $2.50(1.73)$ & $94.32(2.15)$ & $85.17(6.17)$ & $4.12(11.76)$ \\
\hline & $\mathrm{CFS}^{*}$ & 218 & $37.57(18.66)$ & 56.9 & $28.76(8.11)$ & $3.4(10.59)$ & $57.8,22.5,19.7$ & $2.30(1.11)$ & $94.09(3.35)$ & $88.81(7.80)$ & $3.26(12.79)$ \\
\hline & $\mathrm{CHS}$ & 365 & $77.44(4.65)$ & 64.9 & $27.10(4.41)$ & $10.50(15.14)$ & $25.8,39.2,35.1$ & $2.63(1.57)$ & $94.41(1.91)$ & $84.87(5.96)$ & $3.93(11.89)$ \\
\hline & $\mathrm{FHS}^{*}$ & 192 & $57.45(9.68)$ & 51.0 & $28.87(5.16)$ & $7.30(14.38)$ & $38.0,31.8,30.2$ & $2.42(1.51)$ & $94.73(1.80)$ & $85.76(5.46)$ & $2.82(8.38)$ \\
\hline & MrOS & 2181 & $76.65(5.60)$ & 0.0 & $27.21(3.75)$ & $13.00(18.00)$ & $18.9,36.1,45.0$ & $3.54(1.48)$ & $93.85(1.73)$ & $84.39(5.88)$ & $4.40(9.95)$ \\
\hline & WASHS & 1508 & $52.29(13.71)$ & 40.9 & $31.84(7.93)$ & $7.24(15.37)$ & $40.1,31.1,28.8$ & $3.56(2.00)$ & $94.56(2.38)$ & $84.61(7.86)$ & $5.44(13.82)$ \\
\hline \multirow{2}{*}{$\begin{array}{l}\text { Hispanic, Latino- } \\
\text { American }\end{array}$} & $\mathrm{HCHS}, \mathrm{SOL}$ & 7155 & $46.10(13.81)$ & 57.8 & $29.68(5.86)$ & $2.00(6.15)$ & $69.1,19.3,11.6$ & $N, A$ & $96.46(0.95)$ & $87.06(6.11)$ & $0.83(2.99)$ \\
\hline & Starr & 782 & $52.34(11.29)$ & 71.9 & $32.15(6.78)$ & $10.35(17.18)$ & $31.5,31.5,37.1$ & $N, A$ & 94.65 (2.09) & $85.78(7.50)$ & 2.83 (8.79) \\
\hline
\end{tabular}

Eight studies contributed 13,257 individuals with genomic data imputed with a TOPMed Freeze $5 \mathrm{~b}$ reference panel and objectively measured phenotypes (225 African-Americans, 5095 European-Americans, 7937 Hispanic/Latino-Americans). ARIC, CFS, CHS, FHS, and HCHS/SOL imputed genomic data reflect individuals without available sequencing in TOPMed Freeze 6. The overall sample had a mean age of 53.7 and was $46.9 \%$ female. Values are displayed as mean (SD), except for the skewed apnea-hypopnea Index, which is displayed as median (IQR). Sample size N reflects individuals with non-missing AHI and covariate values.

*Family cohort

WatchPAT-200) [33]. Seven hundred eighty-two HA individuals were studied, using Affymetrix 6.0 genotyping data.

The Western Australian Sleep Health Study (WASH S) is a clinic-based study focused on the epidemiology and genetics of SDB [34]. PSG was obtained from 1508 European-ancestry patients (91\% referred for SDB evaluation) from 2006 to 2010 (Compumedics Series E). Genotyping was performed using the Illumina Omni 2.5 array.

Imputed genotype data were available for additional members of the TOPMed cohorts described above. Study/ population combinations with fewer than 100 individuals were excluded. ARIC contributed an additional 631 EA individuals (Affymetrix 6.0; dbGaP phg000035.v1.p1). CFS contributed 225 AA and 218 EA individuals (Affymetrix 6.0; Illumina OmniExpress+Exome, Exome, and IBC). CHS contributed 365 individuals (Illumina CNV370 and IBC; phg000135.v1.p1 and phg000077.v1.p1). FHS contributed 192 EA individuals (Affymetrix $500 \mathrm{k}$; phg000006.v7). HCHS/SOL contributed 7155 HA individuals (Illumina Omni 2.5; phg000663.v1).

\section{Phenotype and covariate definitions}

We examined several SDB measures, including specific measures of OSA: AHI (number of apneas plus hypopneas per hour of sleep, with a minimum 3\% desaturation per event) and average oxyhemoglobin desaturation per apnea or hypopnea, and measures of SDB severity [14]: average and minimum $\mathrm{SpO}_{2}$ and the percentage of the night with $\mathrm{SpO}_{2}<90 \%$ (Per90). Apart from WASHS, all sleep data were scored by blinded scorers at one central Sleep Reading Center with high levels of scorer reliability using well-defined procedures [35]. The AHI reflected all events. We did not attempt to disentangle the apneahypopnea index from central versus obstructive sleep apnea events, due to the relatively low prevalence of central sleep apnea $(<2 \%)$ in these largely community-based studies [36, 37] (some of which are enriched with snorers) and the complexities of classifying mixed events. We adjusted for age, age ${ }^{2}$, sex, age $\times$ sex, body mass index (BMI), and $\mathrm{BMI}^{2}$ due to known age and sex effects, some of which are non-linearly associated with outcomes, and our goal of identifying obesityindependent loci. Age and BMI were obtained at the time of the sleep recording. We adjusted for BMI as over half of the AHI trait heritability is attributable to factors other than obesity as measured by the BMI and our goal was to identify associations with other mechanistic pathways (e.g., ventilatory control) that could indicate novel future targets. Phenotype analyses were pooled within populations to aggregate very rare variants for testing and therefore further adjusted for study. Population assignments were based on self-report, in accordance with other research from TOPMed and other consortia. AsA and EA-identifying individuals with population principal components $>5$ standard deviations [38] from applicable 1000 Genomes and Human Genome Diversity Project super-populations were excluded. We used a two-stage procedure to rank-normalize the phenotypes adjusted for covariates [39]. Cryptic relatedness and population substructure were controlled for using linear mixed models. Genomic control was applied to populationspecific results (or cohort-specific imputed genotype results).

\section{WGS and genotyping}

Sequence data were derived from the TOPMed Freeze 6a release, jointly called by the TOPMed Informatics Research Center at the University of Michigan (http:// github.com/statgen/topmed_variant_calling). The methodology was described elsewhere [12]. In brief, WGS 
was performed at the Broad Institute (ARIC, FHS, MESA), Baylor College of Medicine (ARIC, CHS, $\mathrm{HCHS} / \mathrm{SOL}$ ), and the University of Washington (CFS, JHS). Additional ARIC and HCHS/SOL WGS funded by CCDG (https://www.genome.gov/27563570) and performed at Baylor College of Medicine were included in the jointly called data. TOPMed and CCDG calling pipelines have functionally equivalent outcomes despite data processing differences (as detailed in [40]). WGS data were merged and normalized; inferred sequence contamination was identified; and SNPs and small indels were detected (structural variants are not currently available). Lower quality variants were excluded using Mendelian consistency checks. Variants were aligned to Build 38 and annotated using snpEff $4.3 \mathrm{t}$ [41]. We excluded variants with $<10 \times$ depth or $>5 \%$ missingness, leaving 152.7 million polymorphic variants in 7988 individuals with SDB phenotypes. Up to $22,030,888$ variants from individuals with sequencing were tested in the GWAS analyses, following filtering for quality control and minor allele frequencies.

Genotype data were imputed using the TOPMed Imputation Server [13] using a Freeze 5b (Build 38) template. Forward strand checks were performed using the Strand database and the Haplotype Reference Consortium imputation preparation script (https://www.well.ox. ac.uk/ wrayner/tools/) and confirmed using Ensembl variant allele checks and internal QC performed on the server. Study-level data were imputed separately. Analyses on variants with $r^{2}$ score $>0.5$ were therefore performed separately for each study. Up to $22,105,437$ variants from individuals with imputed data were tested in the GWAS analyses, following filtering for quality control, imputation $\mathrm{r}^{2}$, and minor allele frequencies.

\section{Statistical analyses}

Single and grouped variant analyses were performed using EMMAX and MMSKAT, both within the EPAC TS suite (v3.3) [42]. WGS genetic relatedness matrices (GRM) were constructed using autosomal variants (MAF $>0.1 \%$ ) following a comparison of EPACTS point-wise heritability estimates of the AHI using different minimal MAFs. A grid search identified optimal GRM parameters with imputed data (MAF $>0.5 \%, r^{2}>0.90$ ) using 929 ARIC individuals with imputation and WGS data. $\log _{10}$ $P$-values using identical association test parameters had a Spearman's $\rho$ correlation of 0.951 between WGS and imputed data. Matrices were constructed separately for each study + population combination (due to potentially differential imputation coverage).

Gene-based group sets considered Ensembl-defined non-pseudogenes expressed in any GTEx v7 tissue. Variants needed to clear a series of frequency, regional, functional class, and presumed functionality score filters in order to test a gene using its most biologically plausible variants. Variants could have a maximum minor allele frequency of $5 \%$. Regions were largely exon-based. We also included variants located within experimentally derived promoter regions and Ensembl-derived Tarbase miRNA binding sites; and regulatory variants located within 1000 bases of a particular gene, including ChIPseq determined transcription factor binding sites (TFBS), and Ensembl-derived CTCF, TFBS, and promoter sites [43-45]. Variants from a subset of 19 snpEff gene-based annotation functional classes (e.g., missense or nonsense, but not synonymous mutations) were considered. Finally, group set variants passing these prior filters were additionally filtered for the plausibility of biological function by requiring either a FATHMM-XF score $>0.5$ or a CDTS $<1 \%$ constrained region score $[46,47]$. Exonic variants could alternatively have a PrimateAI score $>0.803$ or a Havrilla et al. $<1 \%$ constrained coding region score $[48,49]$.

Gene-based tests considered variants in WGS-only data. Pooled (across cohort) analyses were performed within each population in order to aggregate information on very rare variants across studies. Combined population results were obtained through meta-analysis of $p$-values weighted by sample size (due to potentially different MAF spectra driven by population demography). A significance level of $p<4.51 \times 10^{-8}$ was used, reflecting a Bonferroni adjustment for all genes tested across all phenotype and population configurations.

A second set-based analysis was designed to query for TFBS annotation enrichment [50]. We performed 250base pair sliding window analyses (to improve power by aggregating additional variants beyond an approximate ChIP-seq peak width of 100 base pairs). We filtered for variants with either a FATHMM-XF score $>0.5$ or a CDTS 1\% score with no MAF cut-offs and metaanalyzed MMSKAT results across the 4 populations, noting windows with $p$-values $<0.01$. These intervals were tested for enrichment of ChIP-seq coordinates with at least $50 \%$ physical overlap for up to 437 transcription factors using ReMap 2018 v1.2 [51].

Single-variant EMMAX tests examined common variants (MAF >0.5\%). Meta-analysis across populations (and imputed genotype studies) used METAL with genomic control [52]. We performed bidirectional discovery and replication using the WGS and imputed samples (noting the high genomic resolution in the WGS samples and the higher sample size in the imputed data). We report results including at least 1000 individuals in discovery analyses, discovery association $p$-values $<1 \times$ $10^{-5}$ and replication association $p$-values $<0.05$. Therefore, no population-specific discovery analyses of Asian-Americans were performed. Multi-ethnic analyses included a minimum of two populations where a variant 
cleared minimum MAF and imputation quality (for chip-based results) criteria. Significance was defined as $p$ $<1 \times 10^{-8}$ in joint analyses, reflecting adjustment for five correlated phenotypes (Additional file 1: Table S3). We performed MetaXcan imputed GTEx gene expression analyses using joint EA results in selected tissues relevant to SDB and GIGSEA pathway analyses of MetaXcan output in whole blood (to maximize power), with empirical $p$-values incorporating 10,000 permutations [53, 54]. Bioinformatics annotations of single-variant results (Additional file 1: Table S7) include significant eQTL associations from GTEx v7, and overlapping promoter and enhancer coordinates derived from Roadmap Epigenomics, BLUEPRINT, and Vermunt et al. brain tissues (enhancers only) [55-58]. Lookups of potentially druggable genes as defined within DGIdb, a database of 56,000 drug-gene interactions from over 30 literature sources, were performed using the GeneCards suite [59, 60].

\section{Results}

\section{Study sample}

A study overview is provided in Additional file 2: Figure S1. Tables 1 and 2 provide a summary of the study samples and SDB traits analyzed using WGS and imputed genotypes, respectively. In total, there were 21,244 individuals (1942 AAs, 229 AsAs, 8341 EAs, and 10732 HAs). Median AHI levels ranged from mildly to moderately elevated, reflecting the age range and sex distribution of each cohort. Pairwise correlations of phenotypes and covariates are provided in Additional file 1: Table S3.

\section{Gene-based results}

Gene-based rare-variant results are presented in Table 3 (for meta-analyzed results across multiple populations) and in Table 4 (for secondary population-specific results). Collectively, we identified four significantly associated genes (Bonferroni $p<4.51 \times 10^{-8}$ ). ARMCX3, identified in the multiple-population analysis, is an Xlinked protein-coding that was associated with average desaturation $\left(p=5.29 \times 10^{-8}\right)$. Two protein-coding genes were identified in population-specific analyses of Per90: MRPS33 $\left(p=1.22 \times 10^{-9}\right)$ and C16orf90 $(p=1.36$ $\left.\times 10^{-8}\right)$. We identified 12 suggestively associated genes $\left(\mathrm{p} \leq 4.22 \times 10^{-7}\right)$. Three genes are druggable $[59,60]$. Nominally significant results $(p<0.01)$ and additional details are presented in Additional file 1: Tables S4 and S5. A list of individual variants comprising each gene is provided in Additional file 1: Table S6.

\section{Single-variant results}

We identified four genome-level significant loci in single-variant analyses (MAF $>0.5 \% ; p<1.0 \times 10^{-8}$; Table 5). In multiple-population analyses, the $2 q 12$ locus (rs77375846; IL18RAP) was associated with average event desaturation in a multiple-population analysis (combined $p=1.57 \times 10^{-9}$ ) and minimum $\mathrm{SpO}_{2}$ (consistent with a previous report [10]). Two novel population-specific loci were identified. The 8p12 locus (rs35447033, NRG1) was associated with AHI in EAs (combined $p=3.02 \times 10^{-9}$, Fig. 1). The 5 p13 locus (rs28777; SLC45A2) was associated with average $\mathrm{SpO}_{2}$ in EAs (combined $p=8.08 \times 10^{-10}$, Fig. 2). In HAs, the 1q32 locus (rs116133558; ATP2B4) was associated with Per90 (combined $p=3.51 \times 10^{-10}$ ) and with average $\mathrm{SpO}_{2}$ (as previously identified [9]). Twelve additional regions were suggestively associated $\left(p<1.0 \times 10^{-7}\right)$. Additional file 1: Table S7 provides additional context for all variants in these loci $\left(p<1.0 \times 10^{-7}\right)$, including imputation quality, significant eQTLs, and overlap with epigenetic regions. Lookups of loci that we have identified in prior publications [9-11] are provided in Additional file 1: Table S8. Manhattan and QQ plots corresponding to the significant associations are provided in Additional file 2: Figures S2-S5. GWAS summary statistics have been posted to the Broad Institute Sleep Disorders Research Portal (https://sleep.hugeamp.org/).

\section{MetaXcan imputed gene expression and GIGSEA pathway analyses}

We used joint WGS and imputed EA results to impute associations with gene expression levels using a MetaXcan framework for six tissues (subcutaneous and visceral omentum adipose, lung, monocytes, skeletal muscle, and whole blood). No individual tests reached Bonferroni significance $\left(p<2.60 \times 10^{-7}\right.$; Additional file 1: Table S9). Genes that were observed in the top 10 results across the varied analyses (Additional file 1: Table S10) included ZNF83 (15 instances) and CHRNE (13 instances).

Whole blood MetaXcan results (with the largest sample size) were further evaluated in GIGSEA-based pathway analyses. KEGG pathway results are shown in Additional file 1: Table S11. The most significantly associated pathway was KEGG_STEROID_HORMONE_BIOSYNTHESIS (average $\mathrm{SpO}_{2}$ empirical $p$-value $\left.=7.00 \times 10^{-4}\right)$. KEGG RIG_I_LIKE_RECEPTOR_SIGNALING_PATHWAY was observed in the top 10 results for four of the five phenotypes. Gene-centric transcription factor binding site (TFBS) enrichment analysis results are presented in Additional file 1: Table S12. V\$PEA3_Q6 (ETV4) was the most significantly associated TFBS (average desaturation empirical $p$-value $=3.00 \times 10^{-4}$ ) and was the strongest association for $\mathrm{AHI}$ and minimum $\mathrm{SpO}_{2}$ (empirical $p$-values 0.002 and 0.001 , respectively). The most significant miRNA binding site enrichment analysis association was GCATTTG,MIR-105 (average $\mathrm{SpO}_{2} p=0.002$; Additional file 1: Table S13). AGGCACT,MIR-515-3P (the strongest AHI association, $p=0.009$ ) was observed in the top ten results for four phenotypes. 
Table 3 Lead gene-based multiple-population results

\begin{tabular}{|c|c|c|c|c|c|c|c|c|c|}
\hline Phenotype & Sex & Gene & B38 positions & $\mathbf{P}$ & $\mathbf{N}$ & Variants & Population P & Population N & $\begin{array}{l}\text { Population } \\
\text { variants }\end{array}$ \\
\hline \multirow[t]{2}{*}{$\begin{array}{l}\text { Avg } \\
\text { desaturation }\end{array}$} & All & ARMCX3 & $\begin{array}{l}X: 101,623,082- \\
101,625,765\end{array}$ & $3.48 \times 10^{-8}$ & 5222 & 41 & $0.220,0.179,2.17 \times 10^{-6}, 8.93 \times 10^{-4}$ & $1545 ; 227 ; 2994 ; 456$ & $8,5,24,9$ \\
\hline & All & ARMCX3-AS1 & $\begin{array}{l}X: 101,623,082- \\
101,625,153\end{array}$ & $3.49 \times 10^{-8}$ & 5222 & 38 & $0.225,0.179,2.19 \times 10^{-6}, 8.20 \times 10^{-4}$ & $1545 ; 227 ; 2994 ; 456$ & $7,5,23,8$ \\
\hline Per90 & All & OR5K2 & $\begin{array}{l}3: 98,497,633- \\
98,498,634\end{array}$ & $2.55 \times 10^{-7}$ & 7986 & 7 & $0.143,0.440,4.14 \times 10^{-2}, 2.74 \times 10^{-7}$ & $1712 ; 229 ; 3,242 ; 2803$ & $4,2,1,1$ \\
\hline Per90 & Females & ZZEF1 & $\begin{array}{l}17: 4,004,409- \\
4,144,018\end{array}$ & $4.22 \times 10^{-7}$ & 4485 & 236 & $0.634,0.337,5.03 \times 10^{-4}, 3.05 \times 10^{-5}$ & $1009 ; 114 ; 1702 ; 1660$ & $85,16,87,131$ \\
\hline
\end{tabular}

Lead MMSKAT gene-based results meta-analyzed across populations within one order of magnitude of significance $\left(p<4.51 \times 10^{-8}\right)$ are shown. Populationspecific information for each gene is displayed in the latter columns for AA, AsA, EA, and HA, respectively. Individual populations varied in the number of polymorphic variants available for testing (e.g., due to singletons or excessively common variants). ARMCX3-AS1 is a RNA gene that is anti-sense to the proteincoding ARMCX3 gene. Full results for genes with $p<0.01$, including Ensembl-derived gene biotypes and descriptions, are provided in Additional file 1: Table S4. A list of individual variants comprising each gene is provided in Additional file 1: Table S6

\section{ChIP-seq transcription factor binding site interval enrichment}

We performed a sliding window analysis to examine enriched intervals containing ChIP-seq derived coordinates for up to 437 transcription factors (Table 6, Additional file 1: Table S14). FOXP2 TFBS were consistently the most enriched for all phenotypes. Other notable transcription factors in the top 5 included EGR1, $K D M 4 B, K D M 6 B$, and TP63. KDM4B and $K D M 6 B$ are druggable $[59,60]$. Leading sliding window results are provided in Additional file 1: Table S15.

\section{Discussion}

Sleep-disordered breathing is associated with increased risk of a wide range of disorders, including cardiometabolic disease, cancer, cognitive impairment, and interstitial lung diseases, as well as premature mortality [2, 61]. Treatment options, however, are limited by a lack of knowledge of molecular pathways, including those that may be "druggable." Recent analyses of SDB traits have focused on common variants and identified several preliminary genomelevel significant associations [9-11], but did not address gene-based or rare-variant effects. Ten studies and over 21, 000 individuals of multiple ancestries with WGS data at unprecedented resolution from the NHLBI TOPMed program combined with densely imputed data from other sources contributed to these results. We identified several variant, gene-based, and pathway-level associations. Analyses adjusted for obesity, a major SDB risk factor, identified loci and genes implicated in pulmonary, inflammatory, and craniofacial pathways. Some associations were populationspecific, while others were sex-specific, consistent with population differences and strong sex differences for SDB $[20,62]$. Notably, across multiple ancestral groups, we identified a set-based rare-variant association $(p=3.48 \times$ $10^{-8}$ ) on chromosome $\mathrm{X}$ with $A R M C X 3$.

Table 4 Lead gene-based population-specific results

\begin{tabular}{|c|c|c|c|c|c|c|c|}
\hline Phenotype & Model & Gene & B38 positions & $\mathbf{N}$ & Variants & Singletons & $\mathbf{P}$ \\
\hline \multirow[t]{13}{*}{ Per90 } & \multirow[t]{2}{*}{$\mathrm{HA}$} & LINC01277 & $6: 142,985,371-143,010,415$ & 2803 & 2 & 0 & $5.02 \times 10^{-8}$ \\
\hline & & OR5K2 & $3: 98,497,633-98,498,634$ & 2803 & 1 & 0 & $2.74 \times 10^{-7}$ \\
\hline & \multirow[t]{2}{*}{ AA females } & S100A16* & $1: 153,607,528-153,616,353$ & 1009 & 1 & 1 & $2.07 \times 10^{-7}$ \\
\hline & & CSMD2-AS1 & $1: 33,867,977-33,885,456$ & 1009 & 1 & 1 & $2.07 \times 10^{-7}$ \\
\hline & \multirow[t]{4}{*}{ EA females } & MRPS33 & $7: 141,006,422-141,014,911$ & 1702 & 9 & 8 & $1.22 \times 10^{-9}$ \\
\hline & & LINC01811 & $3: 34,170,921-34,558,474$ & 1702 & 6 & 5 & $9.71 \times 10^{-8}$ \\
\hline & & $N E L F C D^{*}$ & $20: 58,980,722-58,995,761$ & 1702 & 12 & 10 & $3.32 \times 10^{-7}$ \\
\hline & & $S L C 22 A 8^{*}$ & $11: 62,988,399-63,015,986$ & 1702 & 3 & 3 & $3.58 \times 10^{-7}$ \\
\hline & \multirow[t]{2}{*}{ HA females } & AL 132709.1 & $14: 101,077,452-101,077,578$ & 1660 & 2 & 0 & $1.41 \times 10^{-7}$ \\
\hline & & EPHX4 & $1: 92,029,443-92,063,474$ & 1660 & 12 & 10 & $3.48 \times 10^{-7}$ \\
\hline & \multirow[t]{3}{*}{ HA males } & C16orf90 & $16: 3,493,483-3,496,479$ & 1143 & 6 & 3 & $1.36 \times 10^{-8}$ \\
\hline & & TVP23B & $17: 18,781,270-18,806,714$ & 1143 & 4 & 4 & $2.53 \times 10^{-7}$ \\
\hline & & IPCEF1 & $6: 154,154,536-154,356,890$ & 1143 & 10 & 8 & $4.07 \times 10^{-7}$ \\
\hline
\end{tabular}

Lead MMSKAT gene-based population-specific associations within one order of magnitude of significance $\left(p<4.51 \times 10^{-8}\right)$ are shown. The Variants column indicates the number of filtered polymorphic variants with minor allele frequency $<5 \%$ available for testing, a portion of which were singletons. ${ }^{*}$ Druggable gene $[59,60]$. Full results for genes with $p<0.01$, including descriptions, are provided in Additional file 1: Table S5. A list of individual variants comprising each gene is provided in Additional file 1: Table 56 


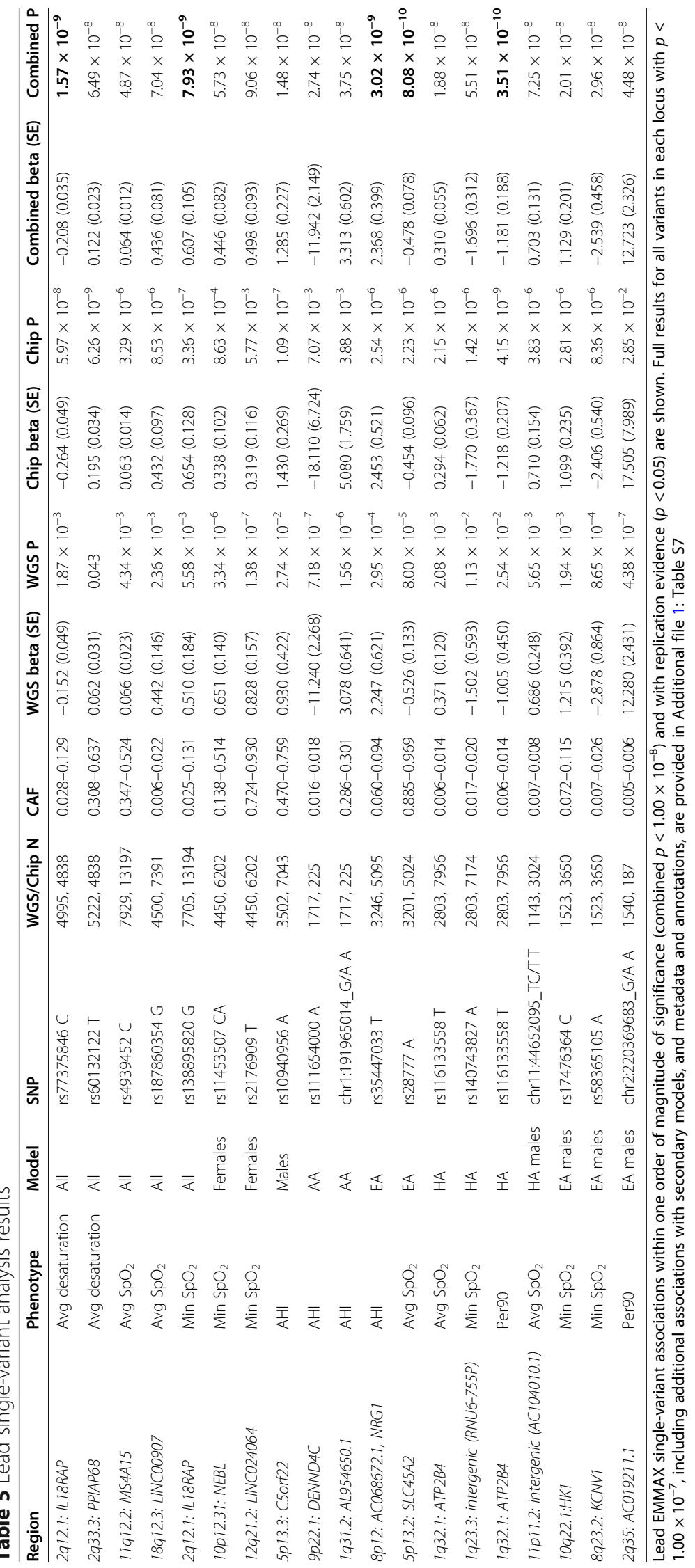




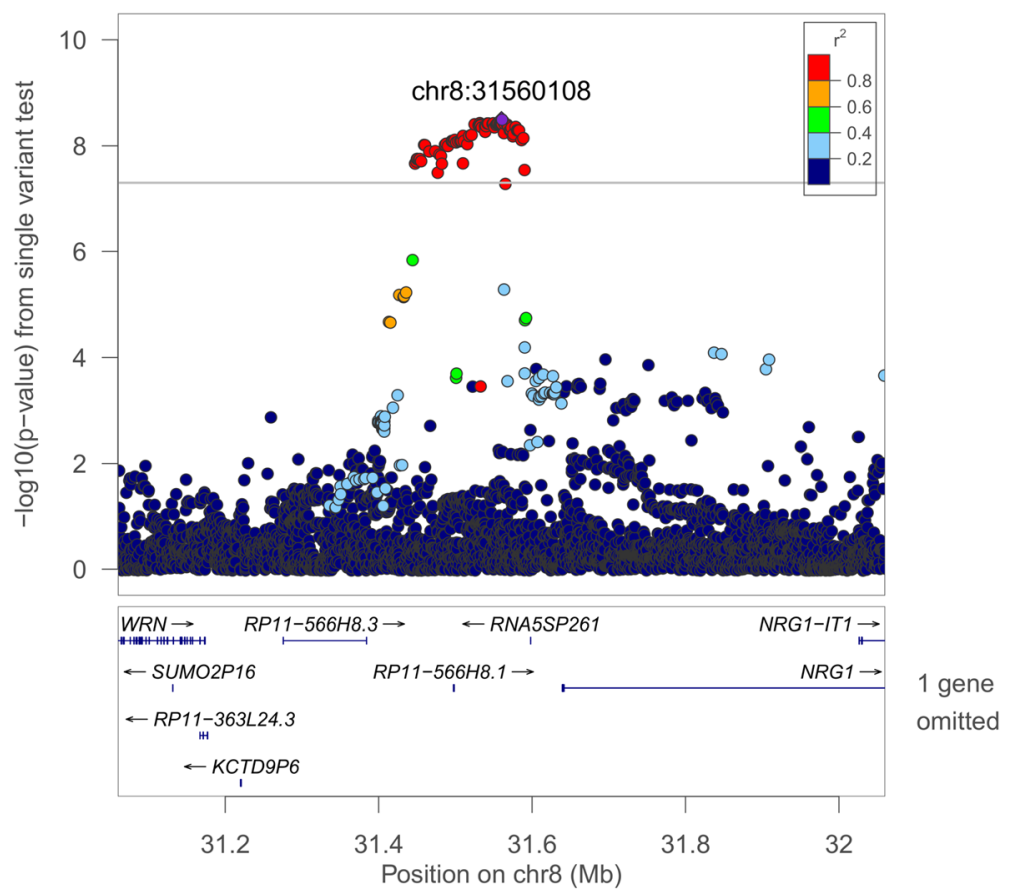

Fig. 1 Regional plot of the rs35447033 association with AHI in European-ancestry individuals. Joint WGS and imputed results are shown, using Build 38 coordinates on the X-axis. Log-transformed $p$-values are shown on the Y-axis. Variant colors indicate the degree of linkage disequilibrium with the lead variant rs35447033

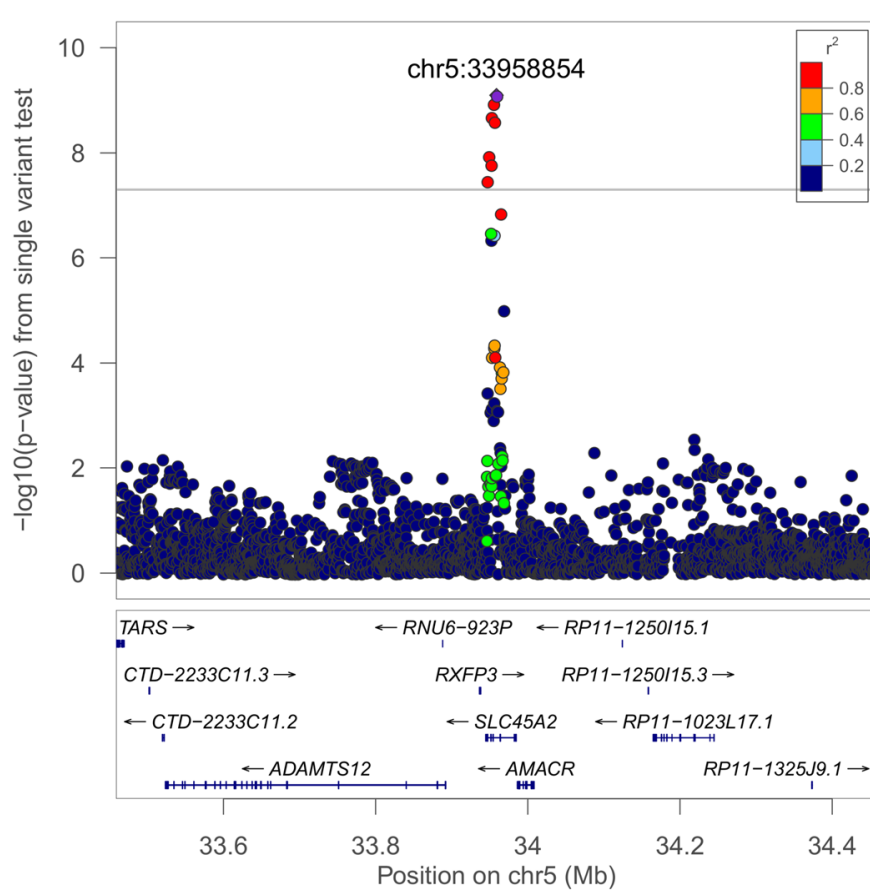

6 genes omitted

Fig. 2 Regional plot of the rs 28777 association with average $\mathrm{SpO}_{2}$ in European-ancestry individuals. Joint WGS and imputed results are shown, using Build 38 coordinates on the X-axis. Log-transformed p-values are shown on the Y-axis. Variant colors indicate the degree of linkage disequilibrium with the lead variant rs28777 
Table 6 Transcription factor binding site interval enrichment results

\begin{tabular}{|c|c|c|c|c|}
\hline Phenotype & Transcription factor & \# Observed overlap & \# Expected overlap & $-\log 10$ (E-value) \\
\hline \multirow[t]{5}{*}{$\mathrm{AHI}$} & FOXP2 & 588 & 36.20 & 473.99 \\
\hline & KDM6B & 630 & 51.58 & 435.29 \\
\hline & THAP1 & 505 & 31.89 & 402.07 \\
\hline & KLF9 & 745 & 91.81 & 395.52 \\
\hline & TP63 & 997 & 182.22 & 383.85 \\
\hline \multirow[t]{5}{*}{ Average desaturation } & FOXP2 & 493 & 22.32 & 460.00 \\
\hline & THAP1 & 439 & 19.55 & 412.76 \\
\hline & UBTF & 489 & 28.20 & 407.50 \\
\hline & TP63 & 788 & 109.36 & 382.89 \\
\hline & $K D M 6 B$ & 482 & 30.98 & 380.39 \\
\hline \multirow[t]{5}{*}{ Average $\mathrm{SpO}_{2}$} & FOXP2 & 582 & 35.87 & 468.89 \\
\hline & KDM6B & 613 & 51.21 & 418.65 \\
\hline & EGR1 & 664 & 66.76 & 404.83 \\
\hline & UBTF & 574 & 46.35 & 399.91 \\
\hline & $K D M 4 B$ & 489 & 29.56 & 398.10 \\
\hline \multirow[t]{5}{*}{$\mathrm{Min} \mathrm{SpO}_{2}$} & FOXP2 & 561 & 35.57 & 445.57 \\
\hline & THAP1 & 515 & 31.32 & 417.89 \\
\hline & KDM6B & 569 & 50.87 & 373.41 \\
\hline & UBTF & 536 & 45.99 & 360.56 \\
\hline & EGR1 & 602 & 66.25 & 346.03 \\
\hline \multirow[t]{5}{*}{ Per90 } & FOXP2 & 689 & 39.05 & 578.42 \\
\hline & $K D M 6 B$ & 739 & 54.79 & 539.69 \\
\hline & TP63 & 1199 & 193.28 & 515.44 \\
\hline & THAP1 & 607 & 34.47 & 509.33 \\
\hline & EGR1 & 786 & 72.09 & 507.27 \\
\hline
\end{tabular}

Two-hundred-fifty-base pair sliding window coordinates with association $p<0.01$ were queried for interval enrichment of ChIP-seq-derived transcription factor binding sites using the ReMap annotation tool. ChIP-seq coordinates were required to have $>50 \%$ overlap with a sliding window interval. ReMap-derived expected overlaps are obtained from the equivalent number of similarly sized random regions. E-value indicates the expected value, with a higher logtransformed value indicating greater enrichment. Full results are provided in Additional file 1: Table S14

\section{Gene-based results}

Across multiple populations, $A R M C X 3$ (ALEX3) and the RNA anti-sense gene $A R M C X 3-A S 1$ were associated with apnea-hypopnea triggered intermittent hypoxia. $A R M C X 3$ regulates mitochondrial aggregation and trafficking in multiple tissues and facilitates neuronal survival and axon regeneration [63-65]. Wnt signaling regulates reactive oxygen species (ROS) generation and ARMCX3-associated mitochondrial aggregation $[64,66]$. Potential mechanisms for further study include sensitized carotid body chemoreflexes, interaction with inflammatory mechanisms, and neuronal dysfunction within respiratory centers. Sleep apnea and reduced ventilatory drive are enriched in individuals with a primary mitochondrial disorder [67]. Mitochondria are an important source of ROS, which modulate the acute hypoxic ventilatory response. Mitochondria impact HIF1A signaling and may contribute to oxygen sensing $[68,69]$.
ROS are required for intermittent hypoxia-induced respiratory long-term facilitation [70]. These effects may mitigate the level of hypoxia resulting from recurrent apneas, or conversely, lead to ventilatory instability, promoting apnea occurrence. Mitochondrial ROS also activate the NLRP3 inflammasome in multiple pulmonary diseases, consistent with an inflammation model that includes our IL18-pathway and HK1 results, ROSrelated proinflammatory responses to lung capillary pressure, and evidence of alveolar epithelial injury/SDB interactions [10, 69, 71-73]. Our findings suggest value in investigating the mechanisms by which $A R M C X 3$ predisposes to SDB, and whether these associations are mediated by neuronal dysfunction and/or ROS and carotid body sensitization, and interact with the inflammasome.

Additional genes were significantly associated in population-specific analyses, including the mitochondrial ribosomal gene MRPS33. Mitoribosomes are responsible 
for the expression of the 13 essential components of the oxidative phosphorylation system, and a majority of the small subunit proteins have been implicated in disease [74]. The expression of several small and large subunit proteins are altered in a hypoxic environment [75]. MRPS33 expression varies with oxygen treatment in COPD [76].

\section{Single-variant results}

We identified four common frequency associated loci, including multiple-population associations with the IL18RAP region. The IL18RAP region has been associated with minimum $\mathrm{SpO}_{2}$ [10], and here we further identify an association with average event desaturation, highlighting a role in an OSA-specific trait. Multiple variants in this region are also GTEx eQTL variants for both interleukin-18 receptor subunits IL18RAP and IL18R1 (Additional file 1: Table S7) and experimental studies support a role for IL18 signaling in mediating this association, possibly through effects of pulmonary inflammation on gas exchange (reviewed in [10]).

We identified three population-specific loci, including two novel associations in individuals of European ancestry (Figs. 1 and 2). Sixty-five variants in the NRG1 region were associated with the AHI $\left(p<1.0 \times 10^{-8}\right.$, Additional file 1 : Table S7). This region was suggestively associated with sleep apnea in a Korean population [77]; however, the lead signals appear to be independent (rs10097555 Korean $p=$ $2.6 \times 10^{-6}$, EA $\left.p=0.91\right)$. NRG1 is associated with lung development and acute lung injury and mediates inflammasome-induced alveolar cell permeability [78-80]. NRG1 promotes accumulation of HIF1A and has increased expression in vascular smooth muscle cells following exposure to intermittent hypoxia [81, 82]. The lead SLC45A2 region variant rs28777 (average $\mathrm{SpO}_{2} p=8.08 \times 10^{-10}$ ) has been associated with multiple traits and is in a splicing regulatory element with extreme population differentiation [83]. An association in the $A T P 2 B 4$ region with average $\mathrm{SpO}_{2}$ in HAs [9] has been extended to a second hypoxemia trait at the same variant $\left(\operatorname{Per} 90 p=3.31 \times 10^{-10}\right)$. This gene is the main cellular membrane calcium pump in erythrocytes and also regulates vascular tone $[84,85]$.

\section{Pathway analyses}

Several gene pathways were identified in EA individuals using imputed gene expression in whole blood (Additional file 1: Table S11). KEGG_RIG_I_LIKE_RECEPTOR_SIGNALING_PATHWAY (retinoic acid-inducible gene Ilike) was the most commonly observed, occurring in the top 10 results for 4 of the 5 phenotypes. This pathway initiates the immune response to RNA virus infection [86], consistent with a role for inflammation at the NRG1 and IL18RAP loci. Steroid hormone biosynthesis (the most significantly associated pathway), PPAR signaling, and metabolism (via "starch and sucrose metabolism") suggest the importance of biological pathways modulating energy homeostasis and balance and metabolic function [87]. In the gene-centric GIGSEA TFBS analysis, V\$PEA3_Q6 (ETV4) was the lead association for three phenotypes. ETV4 influences branching in the developing lung and regulates hypoxia-inducible factor signaling [88, 89], a major mechanism influencing ventilatory control.

\section{Transcription factor binding site enrichment}

Several transcription factors were identified through interval enrichment of observed TFBS across the genome (Table 6). FOXP2 was consistently the most enriched transcription factor and is known to regulate gene expression in epithelial lung tissue and response to lung injury through an inflammatory mechanism $[90,91]$. FOXP2 is also expressed in brainstem respiratory areas including the pre-Bötzinger complex (which is essential for respiratory rhythmogenesis) and impacts airway morphology [92, 93]. Two lysine demethylases $(K D M 4 B$ and $K D M 6 B)$ were also identified. $K D M 6 B(J M J D 3)$ is required for a functional pre-Bötzinger complex [94, 95] and reduced KDM6B protein expression was reported in hypoxic OSA patients [96]. Kdm6b also plays roles in immune function and lung development [9799]. Drosophila $K d m 4 b$ knock-outs have increased sleep [100]. KDM4B (JMJD2B) and KDM6B are both members of the JmjC protein domain family and are regulated by HIF1A, require oxygen as a cofactor, and act as oxygen sensors for chromatin in hypoxia [101, 102]. EGR1 mediates hypoxia-induced pulmonary fibrosis [103]. TP63 is associated with cleft palate in Tp63 deficient mice, which is associated with an increased prevalence of OSA [104, 105], suggesting that its relationship to OSA may be through pathways influencing craniofacial development. Among the leading 250-base pair sliding window results (Additional file 1: Table S15), 4:105708751-105709001 (Per90 HA $p=2.72$ $\times 10^{-9}$ ) is of note due to regional associations with lung function and expression in the human lung [106].

\section{Strengths and weaknesses}

This study is the first genome-wide analysis of objectively measured SDB traits using deep sequencing. Together with improved imputation quality, the TOPMed resource has enabled unprecedented genetic resolution. We examined clinically relevant phenotypes measured using rigorous methodology $[2,14]$. We analyzed data from 10 studies of individuals from four population groups that used different ascertainment strategies, which may potentially improve the generalization of our results. While this analysis is among the largest performed for SDB traits to date, our moderate sample size has lower power to detect weaker associations, and data were not available to replicate these first rare-variant associations. We did not specifically study the central apnea-hypopnea index due to the relatively low prevalence of central sleep apnea $(<2 \%)$ 
in these largely community-based studies [36, 37]. While there are multiple lines of evidence in the literature to support our findings, additional experimental follow-up analyses are required.

\section{Conclusions}

We have identified the first rare-variant and additional common-variant associations at genome-level significance with objectively measured SDB traits in humans. The results point to biologically relevant pathways for further study, including a novel X-linked association (ARMCX3), and a number of associations in genes that modulate lung development, inflammation, respiratory rhythmogenesis, and HIF1A-mediated hypoxic-response pathways. These associations will motivate future sample collection and follow-up in cell-line and animal validation studies, with potential therapeutic benefit for sleep-disordered breathing and related comorbidities.

\begin{abstract}
Abbreviations
AA: African-American; AsA: Asian-American; BMI: Body mass index; CCDG: Centers for Common Disease Genomics; EA: European-American/ Australian; GWAS: Genome-wide association study; HA: Hispanic/LatinoAmerican; MAF: Minor allele frequency; OSA: Obstructive sleep apnea; Per90: Percent of the sleep recording with oxyhemoglobin saturation < 90\%; ROS: Reactive oxygen species; SDB: Sleep-disordered breathing; $\mathrm{SpO}_{2}$ : Oxyhemoglobin saturation; TFBS: Transcription factor binding site; TOPMed: Trans-Omics for Precision Medicine; WGS: Whole-genome sequencing
\end{abstract}

\section{Supplementary Information}

The online version contains supplementary material available at https:/doi. org/10.1186/s13073-021-00917-8.

\section{Additional file 1: Table S1. NHLBI TOPMed Consortium. Table S2.} NHLBI TOPMed Consortium Sleep Working Group. Table S3. Pairwise Phenotype and Covariate Correlations. Table S4. MMSKAT gene-based multiple-population results $(p<0.01)$. Table S5. MMSKAT gene-based population-specific results $(p<0.01)$. Table $\mathbf{S 6}$. Lead MMSKAT result variants. Table S7. Single-variant analysis results for lead loci. Table S8. Lookups of previously reported GWAS results. Table S9. MetaXcan imputed gene expression results. Table S10. Lead genes in multiple MetaXcan results. Table S11. GIGSEA KEGG pathway results. Table S12. GIGSEA MsigDB transcription factor binding site enrichment results. Table S13. GIGSEA MsigDB miRNA binding site enrichment results. Table S14. Sliding window analysis transcription factor binding analysis enrichment. Table S15. Lead sliding window analysis results.

Additional file 2: Figure S1. Study Overview. Figure S2. NRG1 Locus Models Manhattan and QQ Plots. Figure S3. SLC45A2 Locus Models Manhattan and QQ Plots. Figure S4. IL18RAP Locus Models Manhattan and QQ Plots. Figure S5. ATP2B4 Locus Models Manhattan and QQ Plots.

\section{Acknowledgements}

We acknowledge our TOPMed Consortium and TOPMed Sleep Traits Working Group collaborators, who are listed in Additional file 1: Tables S1 and S2. The authors wish to thank the participants and study staff of all of our cohorts for their important contributions. We gratefully acknowledge the studies and participants who provided biological samples and data for TOPMed. The authors thank the staff and participants of the ARIC study for their important contributions. A full list of principal CHS investigators and institutions can be found at CHS-NHLBl.org. The Framingham Heart Study thanks the study participants and the multitude of investigators who over its 70-year history continue to contribute so much to further our knowledge of heart, lung, blood, and sleep disorders and associated traits. The authors thank the staff and participants of $\mathrm{HCHS} / \mathrm{SOL}$ for their important contributions. The views expressed in this manuscript are those of the authors and do not necessarily represent the views of the National Heart, Lung, and Blood Institute; the National Institutes of Health; or the US Department of Health and Human Services. This manuscript was not approved by the HCHS/SOL publications committee. Investigator's website-http://www.cscc. unc.edu/hchs/. The authors also wish to thank the staff and participants of the JHS. We thank the field staff in Starr County for their careful collection of these data and are especially grateful to the participants who so graciously cooperated and gave of their time.

\section{Authors' contributions}

Conception and design: B.E.C., E.A.B., A.C., L.A.C., R.C.K., K.E.N., B.M.P., J.I.R., S.S.R., R.P.T., R.S.V., J.G.W., and S.R. Data acquisition: B.E.C., J.L., T.S., M.Z., H.C., S.A.G., D.J.G., J.M.L., J.L., X.L., H.M., S.R.P., S.M.P., R.S., N.A.S., H.W., X.Z., D.S.E., C.L.H., D.R.H., S.M., L.J.P., K.L.S., G.J.T., and S.R. Analysis: all authors.

Interpretation, draft and review, and final approval: all authors. B.E.C. and S.R. had full access to the study data and take responsibility for the integrity of the data and accuracy of analyses.

\section{Funding}

Whole-genome sequencing (WGS) for the Trans-Omics in Precision Medicine (TOPMed) program was supported by the National Heart, Lung, and Blood Institute (NHLBI). WGS for "NHLBI TOPMed: Trans-Omics for Precision Medicine Whole Genome Sequencing Project: ARIC" (phs001211.v1.p1) was performed at Baylor College of Medicine Human Genome Sequencing Center (HHSN268201500015C and 3U54HG003273-12S2) and the Broad Institute of MIT and Harvard (3R01HL092577-06S1). WGS for "NHLBI TOPMed: The Cleveland Family Study (WGS)" (phs000954.v2.p1) was performed at the University of Washington Northwest Genomics Center (3R01HL098433-05S1). WGS for "NHLBI TOPMed: Cardiovascular Health Study" (phs001368.v1.p1) was performed at Baylor College of Medicine Human Genome Sequencing Center (HHSN268201500015C). WGS for "NHLBI TOPMed: Whole Genome Sequencing and Related Phenotypes in the Framingham Heart Study" (phs000974.v3.p2) was performed at the Broad Institute of MIT and Harvard (3R01HL092577-06S1). WGS for "NHLBI TOPMed: Hispanic Community Health Study/Study of Latinos (HCHS/SOL)" (phs001395) was performed at the Baylor College of Medicine Human Genome Sequencing Center (HHSN268201500015C and 3U54HG003273-12S2). WGS for "NHLBI TOPMed: The Jackson Heart Study" (phs000964.v3.p1) was performed at the University of Washington Northwest Genomics Center (HHSN268201100037C). WGS for "NHLBI TOPMed: NHLBI TOPMed: MESA" (phs001416.v1.p1) was performed at the Broad Institute of MIT and Harvard (3U54HG003067-13S1). Centralized read mapping and genotype calling, along with variant quality metrics and filtering were provided by the TOPMed Informatics Research Center (3RO1HL117626-02S1). Phenotype harmonization, data management, sample-identity QC, and general study coordination were provided by the TOPMed Data Coordinating Center (3R01HL-120393-02S1).

The Genome Sequencing Program (GSP) was funded by the National Human Genome Research Institute (NHGRI); the National Heart, Lung, and Blood Institute (NHLBI); and the National Eye Institute (NEI). The GSP Coordinating Center (U24 HG008956) contributed to cross-program scientific initiatives and provided logistical and general study coordination. The Centers for Common Disease Genomics (CCDG) program was supported by NHGRI and $\mathrm{NHLBI}$, and CCDG-funded whole-genome sequencing of the ARIC and HCHS/SOL studies was performed at the Baylor College of Medicine Human Genome Sequencing Center (UM1 HG008898 and R01HL059367). Brian Cade is supported by grants from the National Institutes of Health [K01-HL135405-01, R01-HL113338-04, R35-HL135818-01] and the American Thoracic Society Foundation (http://foundation.thoracic.org). Susan Redline is partially supported by grants from the National Institutes of Health [R35HL135818-01, R01-HL113338-04]. Sanjay Patel has had grant support through his institution from the ResMed Foundation, the American Sleep Medicine Foundation, Bayer Pharmaceuticals, and Philips Respironics. James Wilson is supported by U54GM115428 from the National Institute of General Medical Sciences.

The Atherosclerosis Risk in Communities (ARIC) study has been funded in whole or in part with Federal funds from the National Heart, Lung, and Blood Institute, National Institutes of Health, Department of Health and 
Human Services (contract numbers HHSN2682017000011, HHSN268201700002I, HHSN268201700003I, HHSN268201700004I, and HHSN268201700005I), R01HL087641, R01HL059367, and R01HL086694; National Human Genome Research Institute contract U01HG004402; and National Institutes of Health contract HHSN268200625226C. Infrastructure was partly supported by Grant Number UL1RR025005, a component of the National Institutes of Health and NIH Roadmap for Medical Research. This Cardiovascular Health Study (CHS) research was supported by NHLBI contracts HHSN268201200036C, HHSN268200800007C, HHSN268200960009C, HHSN268201800001C N01HC55222, N01HC85079, N01HC85080, N01HC85081, N01HC85082, N01HC85083, and N01HC85086 and NHLBI grants U01HL080295, U01HL130114, R01HL087652, R01HL105756, R01HL103612, R01HL085251, and R01HL120393 with additional contribution from the National Institute of Neurological Disorders and Stroke (NINDS). Additional support was provided through R01AG023629 from the National Institute on Aging (NIA).

The Cleveland Family Study has been supported by National Institutes of Health grants [R01-HL046380, KL2-RR024990, R35-HL135818, and R01-HL113338]. The Framingham Heart Study (FHS) has been supported by contracts N01HC-25195 and HHSN268201500001I and grant R01 HL092577.

The Hispanic Community Health Study/Study of Latinos was carried out as a collaborative study supported by contracts from the NHLBI to the University of North Carolina (HHSN268201300001//N01-HC65233), University of Miami (HHSN268201300004I/N01-HC65234), Albert Einstein College of Medicine (HHSN2682013000021/N01-HC65235), University of Illinois at Chicago (HHSN268201300003I), Northwestern University (N01-HC65236), and San Diego State University (HHSN2682013000051/N01-HC65237). The following Institutes/Centers/Offices contribute to the HCHS/SOL through a transfer of funds to the NHLBI: National Institute on Minority Health and Health Disparities, National Institute on Deafness and Other Communication Disorders, National Institute of Dental and Craniofacial Research, National Institute of Diabetes and Digestive and Kidney Diseases, National Institute of Neurological Disorders and Stroke, and NIH Institution-Office of Dietary Supplements. The Genetic Analysis Center at Washington University was supported by NHLBI and NIDCR contracts (HHSN268201300005C AM03 and MOD03).

The Jackson Heart Study (JHS) is supported and conducted in collaboration with Jackson State University (HHSN268201800013l), Tougaloo College (HHSN268201800014l), the Mississippi State Department of Health (HHSN2682018000151/HHSN26800001) and the University of Mississippi Medical Center (HHSN268201800010l, HHSN2682018000111, and HHSN268201800012l) contracts from the National Heart, Lung, and Blood Institute (NHLBI) and the National Institute for Minority Health and Health Disparities (NIMHD).

MESA and the MESA SHARe project are conducted and supported by the National Heart, Lung, and Blood Institute (NHLBI) in collaboration with MESA investigators. Support for MESA is provided by contracts 75N92020D00001, HHSN268201500003I, N01-HC-95159, 75N92020D00005, N01-HC-95160, 75N92020D00002, NO1-HC-95161, 75N92020D00003, NO1-HC95162, 75N92020D00006, N01-HC-95163, 75N92020D00004, N01-HC-95164, 75N92020D00007, N01-HC-95165, N01-HC-95166, N01-HC-95167, N01-HC95168, N01-HC-95169, UL1-TR-000040, UL1-TR-001079, UL1-TR-001420. Funding for SHARe genotyping was provided by NHLBI Contract N02-HL-64278. Genotyping was performed at Affymetrix (Santa Clara, California, USA) and the Broad Institute of Harvard and MIT (Boston, Massachusetts, USA) using the Affymetrix Genome-Wide Human SNP Array 6.0. The provision of genotyping data was supported in part by the National Center for Advancing Translational Sciences, CTSI grant UL1TR001881, and the National Institute of Diabetes and Digestive and Kidney Disease Diabetes Research Center (DRC) grant DK063491 to the Southern California Diabetes Endocrinology Research Center.

The Osteoporotic Fractures in Men (MrOS) Study is supported by NIH funding. The following institutes provide support: the National Institute on Aging (NIA), the National Institute of Arthritis and Musculoskeletal and Skin Diseases (NIAM S), NCATS, and NIH Roadmap for Medical Research under the following grant numbers: U01 AG027810, U01 AG042124, U01 AG042139, U01 AG042140, U01 AG042143, U01 AG042145, U01 AG042168, U01 AR066160, and UL1 TR000128. The NHLBI provides funding for the MrOS Sleep ancillary study "Outcomes of Sleep Disorders in Older Men" under the following grant numbers: R01 HL071194, R01 HL070848, R01 HL070847, R01 HL070842, R01 HL070841, R01 HL070837, R01 HL070838, and R01 HL070839. The NIAMS provides funding for the MrOS ancillary study "Replication of candidate gene associations and bone strength phenotype in MrOS" under the grant number R01 AR051124. The NIAMS provides funding for the MrOS ancillary study "GWAS in MrOS and SOF" under the grant number RC2 AR058973.

The Starr County Health Studies is supported in part by grants R01 DK073541, U01 DK085501, R01 Al085014, and R01 HL102830 from the National Institutes of Health, and funds from the University of Texas Health Science Center at Houston.

Funding for the Western Australian Sleep Health Study was obtained from the Sir Charles Gairdner and Hollywood Private Hospital Research Foundations, the Western Australian Sleep Disorders Research Institute, and the Centre for Genetic Epidemiology and Biostatistics at the University of Western Australia. Funding for the GWAS genotyping was obtained from the Ontario Institute for Cancer Research and a McLaughlin Centre Accelerator Grant from the University of Toronto.

\section{Availability of data and materials}

Variant-level meta-analysis data are available for visualization and download at the Sleep Disorders Knowledge Portal: https://sleep.hugeamp.org/.

\section{Declarations}

\section{Ethics approval and consent to participate}

This study was approved by the Mass General Brigham Institutional Review Board (IRB) (2010P001765) and the Institutional Review Boards from the institutions of all participating studies. All patients have given their written informed consent. The ARIC study was approved by the IRBs of the University of North Carolina at Chapel Hill, Univerity of Minnesota, and Johns Hopkins University. Cleveland Family Study was approved by the IRBs of Case Western Reserve University and Mass General Brigham (formerly Partners HealthCare). The CHS study was approved by the IRBs [or ethics review committee] of University Washington, University of Pittsburgh, and University of California, Sacramento. The Framingham Heart Study was approved by the IRB of the Boston University Medical Center. The JHS study was approved by Jackson State University, Tougaloo College, and the University of Mississippi Medical Center IRBs. The HCHS/SOL study was approved by the IRBs at each field center, and by the Non-Biomedical IRB at the University of North Carolina at Chapel Hill, to the HCHS/SOL Data Coordinating Center. All IRBs approving the study are Non-Biomedical IRB at the University of North Carolina at Chapel Hill, Chapel Hill, NC; Einstein IRB at the Albert Einstein College of Medicine of Yeshiva University, Bronx, NY; IRB at Office for the Protection of Research Subjects (OPRS), University of Illinois at Chicago, Chicago, IL; Human Subject Research Office, University of Miami, Miami, FL; and Institutional Review Board of San Diego State University, San Diego, CA. The MESA study was approved by the IRBs at The Lundquist Institute (formerly Los Angeles BioMedical Research Institute) at Harbor-UCLA Medical Center, University of Washington, Wake Forest School of Medicine, Northwestern University, University of Minnesota, Columbia University, and Johns Hopkins University. The MrOS study was approved by the IRBs of the San Francisco Coordinating center (UCSF and California Pacific Medical Center), University of Alabama at Birmingham, University of Minnesota, Stanford University, University of Pittsburgh, Oregon Health and Science University, and University of California, San Diego. The Starr County study was approved by the IRB at the University of Texas Health Science Center at Houston. The WASHS study was approved by the Human Research Ethics Committee of Sir Charles Gairdner Hospital, as well as The University of Western Australia, the Confidentiality of Health Information Committee, and the Human Research Ethics Committee of the Western Australian Department of Health. The research was conducted in strict compliance with the Declaration of Helsinki.

\section{Consent for publication}

Not applicable.

\section{Competing interests}

The authors disclose the following industry funding, which they believe is unrelated to this study. SRP has had grant support through his institution from the ResMed Foundation, Bayer Pharmaceuticals, and Philips Respironics. BMP serves on the Steering Committee of the Yale Open Data Access Project, funded by Johnson \& Johnson. KLS has grant funding from Merck. The remaining authors declare that they have no competing interests. 


\section{Author details}

'Division of Sleep and Circadian Disorders, Brigham and Women's Hospital, Harvard Medical School, 221 Longwood Avenue, Boston, MA 02115, USA. ${ }^{2}$ Division of Sleep Medicine, Harvard Medical School, Boston, MA 02115, USA. ${ }^{3}$ Program in Medical and Population Genetics, Broad Institute, Cambridge, MA 02142, USA. ${ }^{4}$ Department of Medicine, University of Maryland School of Medicine, Baltimore, MD 21201, USA. ${ }^{5}$ Human Genetics Center, Department of Epidemiology, Human Genetics and Environmental Sciences, School of Public Health, The University of Texas Health Science Center at Houston, Houston, TX 77030, USA. ${ }^{6}$ Center for Precision Health, School of Public Health and School of Biomedical Informatics, The University of Texas Health Science Center at Houston, Houston, TX 77030, USA. ${ }^{7}$ Computational Medicine Core, Center for Lung Biology, UW Medicine Sleep Center, Division of Pulmonary, Critical Care and Sleep Medicine, University of Washington, Seattle, WA 98195, USA. ${ }^{8}$ VA Boston Healthcare System, Boston, MA 02132, USA. ${ }^{9}$ The Institute for Translational Genomics and Population Sciences, Department of Pediatrics, The Lundquist Institute for Biomedical Innovation at Harbor-UCLA Medical Center, Torrance, CA 90502, USA. ${ }^{10}$ Center for Genomic Medicine and Department of Anesthesia, Pain, and Critical Care Medicine, Massachusetts General Hospital, Boston, MA 02114, USA.

${ }^{11}$ Department of Population and Quantitative Health Sciences, School of Medicine, Case Western Reserve University, Cleveland, OH 44106, USA. ${ }^{12}$ Department of Biostatistics, Harvard T.H. Chan School of Public Health, Boston, MA 02115, USA. ${ }^{13}$ Department of Data Science, University of Mississippi Medical Center, Jackson, MS 29216, USA. ${ }^{14}$ Division of Pulmonary, Allergy, and Critical Care Medicine, University of Pittsburgh, Pittsburgh, PA 15213, USA. ${ }^{15}$ Division of Pulmonary, Critical Care and Sleep Medicine, Icahn School of Medicine at Mount Sinai, New York, NY 10029, USA. ${ }^{16}$ California Pacific Medical Center Research Institute, San Francisco, CA 94107, USA. ${ }^{17}$ Department of Pulmonary Physiology and Sleep Medicine, Sir Charles Gairdner Hospital, Perth, Western Australia 6009, Australia. ${ }^{18}$ Sleep Health Service, Respiratory and Sleep Services, Southern Adelaide Local Health Network, Adelaide, South Australia, Australia. ${ }^{19}$ Adelaide Institute for Sleep Health, Flinders University, Adelaide, South Australia, Australia. ${ }^{20}$ School of Public Health, University of Adelaide, Adelaide, South Australia 5000, Australia. ${ }^{21}$ Department of Biostatistics and Center for Statistical Genetics, University of Michigan School of Public Health, Ann Arbor, MI 48109, USA. ${ }^{22}$ Human Genome Sequencing Center, Baylor College of Medicine, Houston, TX 77030, USA. ${ }^{23}$ Department of Medicine, University of Mississippi Medical Center, Jackson, MS 39216, USA. ${ }^{24}$ Jackson Heart Study, Jackson, MS 39216, USA. ${ }^{25}$ Department of Biostatistics, Boston University School of Public Health, Boston, MA 02118, USA. ${ }^{26}$ Framingham Heart Study, Framingham, MA 01702, USA. ${ }^{27}$ Department of Epidemiology and Population Health, Albert Einstein College of Medicine, Bronx, New York 10461, USA. ${ }^{28}$ Department of Genome Sciences, University of Washington, Seattle, WA 98195, USA. ${ }^{29}$ Northwest Genomics Center, Seattle, WA 98105, USA. ${ }^{30}$ Department of Epidemiology and Carolina Center of Genome Sciences, University of North Carolina, Chapel Hill, NC 27514, USA. ${ }^{31}$ Cardiovascular Health Study, Departments of Medicine, Epidemiology, and Health Services, University of Washington, Seattle, WA 98101, USA. ${ }^{32}$ Kaiser Permanente Washington Health Research Institute, Seattle, WA 98101, USA. ${ }^{33}$ Center for Public Health Genomics, University of Virginia, Charlottesville, VA 22908, USA. ${ }^{34}$ Department of Pathology, University of Vermont, Colchester, VT 05405, USA. ${ }^{35}$ Sections of Preventive Medicine and Epidemiology and Cardiology, Department of Medicine, Boston University School of Medicine, Boston, MA 02118, USA. ${ }^{36}$ Department of Epidemiology, Boston University School of Public Health, Boston, MA 02118, USA. ${ }^{37}$ Department of Physiology and Biophysics, University of Mississippi Medical Center, Jackson, MS 39216, USA. ${ }^{38}$ Division of Pulmonary, Critical Care, and Sleep Medicine, Beth Israel Deaconess Medical Center, Boston, MA 02215, USA.

Received: 7 January 2020 Accepted: 28 May 2021

Published online: 26 August 2021

\section{References}

1. Lévy P, Kohler M, McNicholas WT, Barbé F, McEvoy RD, Somers VK, et al. Obstructive sleep apnoea syndrome. Nat Rev Dis Primer. 2015;1 (1):15015. https://doi.org/10.1038/nrdp.2015.15.

2. Peppard PE, Hagen EW. The last 25 years of obstructive sleep apnea epidemiology — and the next 25? Am J Respir Crit Care Med. 2018;197(3): 310-2. https://doi.org/10.1164/rccm.201708-1614PP.
3. Benjafield AV, Ayas NT, Eastwood PR, Heinzer R, IP MSM, Morrell MJ, et al. Estimation of the global prevalence and burden of obstructive sleep apnoea: a literature-based analysis. Lancet Respir Med. 2019;7(8):687-98. https://doi.org/10.1016/S2213-2600(19)30198-5.

4. Patel SR, Larkin EK, Redline S. Shared genetic basis for obstructive sleep apnea and adiposity measures. Int J Obes. 2008;32(5):795-800. https://doi. org/10.1038/sj.ijo.0803803.

5. Liang J, Cade BE, Wang H, Chen H, Gleason K, Larkin EK, et al. Comparison of heritability estimation and linkage analysis for multiple traits using principal component analyses. Genet Epidemiol. 2016;40(3):222-32. https:// doi.org/10.1002/gepi.21957.

6. Wang H, Cade BE, Sofer T, Sands SA, Chen H, Browning SR, et al. Admixture mapping identifies novel loci for obstructive sleep apnea in Hispanic/Latino Americans. Hum Mol Genet. 2019;28(4):675-87. https://doi.org/10.1093/ $\mathrm{hmg} / \mathrm{ddy} 387$.

7. Redline S. Genetics of obstructive sleep apnea. In Principles and practice of sleep medicine, W.C. Kryger Meir H; Roth, Thomas; Dement, ed. St. Louis: Saunders; 2011. p. 1183-93.

8. Eckert DJ, Jordan AS, Merchia P, Malhotra A. Central sleep apnea: pathophysiology and treatment. Chest. 2007;131(2):595-607. https://doi. org/10.1378/chest.06.2287.

9. Cade BE, Chen H, Stilp AM, Gleason K, Sofer T, Ancoli-Israel S, et al. Genetic associations with obstructive sleep apnea traits in Hispanic/Latino Americans. Am J Respir Crit Care Med. 2016;194(7):886-97. https://doi.org/1 0.1164/rccm.201512-24310C

10. Cade BE, Chen H, Stilp AM, Louie T, Ancoli-lsrael S, Arens R, et al. Associations of variants in the hexokinase 1 and interleukin 18 receptor regions with oxyhemoglobin saturation during sleep. PLoS Genet. 2019; 15(4):e1007739. https://doi.org/10.1371/journal.pgen.1007739.

11. Chen H, Cade BE, Gleason K, Bjonnes AC, Stilp AM, Sofer T, et al. Multiethnic meta-analysis identifies RAl1 as a possible obstructive sleep apnea-related quantitative trait locus in men. Am J Respir Cell Mol Biol. 2018;58(3):391-401. https://doi.org/10.1165/rcmb.2017-02370C.

12. Taliun D, Harris DN, Kessler MD, Carlson J, Szpiech ZA, Torres R, et al. Sequencing of 53,831 diverse genomes from the NHLBI TOPMed Program. Nature. 2021;590(7845):290-9. https://doi.org/10.1038/s41586-021-03205-y.

13. Das S, Forer L, Schönherr S, Sidore C, Locke AE, Kwong A, et al. Nextgeneration genotype imputation service and methods. Nat Genet. 2016; 48:1284-7.

14. Kendzerska T, Gershon AS, Hawker G, Leung RS, Tomlinson G. Obstructive sleep apnea and risk of cardiovascular events and all-cause mortality: a decade-long historical cohort study. PLoS Med. 2014;11(2):e1001599. https:// doi.org/10.1371/journal.pmed.1001599.

15. The ARIC Investigators. The Atherosclerosis Risk in Communities (ARIC) Study: design and objectives. The ARIC investigators. Am J Epidemiol. 1989; 129(4):687-702. https://doi.org/10.1093/oxfordjournals.aje.a115184.

16. Fried LP, Borhani NO, Enright P, Furberg CD, Gardin JM, Kronmal RA, et al. The Cardiovascular Health Study: design and rationale. Ann Epidemiol. 1991; 1(3):263-76. https://doi.org/10.1016/1047-2797(91)90005-W.

17. Feinleib M. The Framingham Study: sample selection, follow-up, and methods of analyses. Natl Cancer Inst Monogr. 1985;67:59-64.

18. Quan SF, Howard BV, Iber C, Kiley JP, Nieto FJ, O'Connor GT, et al. The Sleep Heart Health Study: design, rationale, and methods. Sleep. 1997: 20(12):1077-85

19. Bild DE, Bluemke DA, Burke GL, Detrano R, Diez Roux AV, Folsom AR, et al. Multi-Ethnic Study of Atherosclerosis: objectives and design. Am J Epidemiol. 2002;156(9):871-81. https://doi.org/10.1093/aje/kwf113.

20. Chen X, Wang R, Zee P, Lutsey PL, Javaheri S, Alcántara C, et al. Racial/ethnic differences in sleep disturbances: the Multi-Ethnic Study of Atherosclerosis (MESA). Sleep. 2015;38(6):877-88. https://doi.org/10.5665/sleep.4732.

21. Redline S, Tishler PV, Tosteson TD, Williamson J, Kump K, Browner I, et al. The familial aggregation of obstructive sleep apnea. Am J Respir Crit Care Med. 1995;151(3_pt_1):682-7. https://doi.org/10.1164/ajrccm/151.3_Pt_1.682.

22. Sorlie PD, Avilés-Santa LM, Wassertheil-Smoller S, Kaplan RC, Daviglus ML, Giachello AL, et al. Design and implementation of the Hispanic Community Health Study/Study of Latinos. Ann Epidemiol. 2010;20(8):629-41. https:// doi.org/10.1016/j.annepidem.2010.03.015.

23. Redline S, Sotres-Alvarez D, Loredo J, Hall M, Patel SR, Ramos A, et al. Sleepdisordered breathing in Hispanic/Latino individuals of diverse backgrounds. The Hispanic Community Health Study/Study of Latinos. Am J Respir Crit Care Med. 2014;189:335-44. 
24. Westbrook PR, Levendowski DJ, Cvetinovic M, Zavora T, Velimirovic V, Henninger D, et al. Description and validation of the apnea risk evaluation system: a novel method to diagnose sleep apnea-hypopnea in the home. Chest. 2005;128(4):2166-75. https://doi.org/10.1378/chest.128.4.2166.

25. Taylor HA, Wilson JG, Jones DW, Sarpong DF, Srinivasan A, Garrison RJ, et al. Toward resolution of cardiovascular health disparities in African Americans: design and methods of the Jackson Heart Study. Ethn Dis. 2005;15:S6-4-17.

26. Johnson DA, Guo N, Rueschman M, Wang R, Wilson JG, Redline S. Prevalence and correlates of obstructive sleep apnea among African Americans: the Jackson Heart Sleep Study. Sleep. 2018;41(10). https://doi. org/10.1093/sleep/zsy154.

27. Ng SSS, Chan T-O, To K-W, Ngai J, Tung A, Ko FWS, et al. Validation of Embletta portable diagnostic system for identifying patients with suspected obstructive sleep apnoea syndrome (OSAS). Respirol Carlton Vic. 2010;15: 336-42.

28. Orwoll E, Blank JB, Barrett-Connor E, Cauley J, Cummings S, Ensrud K, et al. Design and baseline characteristics of the osteoporotic fractures in men (MrOS) study-a large observational study of the determinants of fracture in older men. Contemp Clin Trials. 2005;26(5):569-85. https://doi.org/10.1016/j. cct.2005.05.006.

29. Blank JB, Cawthon PM, Carrion-Petersen ML, Harper L, Johnson JP, Mitson E, et al. Overview of recruitment for the osteoporotic fractures in men study (MrOS). Contemp Clin Trials. 2005;26(5):557-68. https://doi.org/10.1016/j.cct.2 005.05.005.

30. Mehra R, Stone KL, Blackwell T, Ancoli Israel S, Dam T-TL, Stefanick ML, et al. Prevalence and correlates of sleep-disordered breathing in older men: osteoporotic fractures in men sleep study. J Am Geriatr Soc. 2007;55(9): 1356-64. https://doi.org/10.1111/j.1532-5415.2007.01290.x.

31. Hanis CL, Ferrell RE, Barton SA, Aguilar L, Garza-lbarra A, Tulloch BR, et al. Diabetes among Mexican Americans in Starr County, Texas. Am J Epidemiol. 1983;118(5):659-72. https://doi.org/10.1093/oxfordjournals.aje.a113677.

32. Hanis $\mathrm{CL}$, Redline $\mathrm{S}$, Cade BE, Bell Gl, Cox NJ, Below JE, et al. Beyond type 2 diabetes, obesity and hypertension: an axis including sleep apnea, left ventricular hypertrophy, endothelial dysfunction, and aortic stiffness among Mexican Americans in Starr County, Texas. Cardiovasc Diabetol. 2016;15(1): 86. https://doi.org/10.1186/s12933-016-0405-6.

33. Choi JH, Kim EJ, Kim YS, Choi J, Kim TH, Kwon SY, et al. Validation study of portable device for the diagnosis of obstructive sleep apnea according to the new AASM scoring criteria: Watch-PAT 100. Acta Otolaryngol (Stockh.). 2010;130(7):838-43. https://doi.org/10.3109/00016480903431139.

34. Mukherjee S, Hillman D, Lee J, Fedson A, Simpson L, Ward K, et al. Cohort profile: the Western Australian Sleep Health Study. Sleep Breath. 2012;16(1): 205-15. https://doi.org/10.1007/s11325-011-0491-3.

35. Redline S, Sanders MH, Lind BK, Quan SF, Iber C, Gottlieb DJ, et al. Methods for obtaining and analyzing unattended polysomnography data for a multicenter study. Sleep Heart Health Research Group. Sleep. 1998;21:759-67.

36. Donovan LM, Kapur VK. Prevalence and characteristics of central compared to obstructive sleep apnea: analyses from the Sleep Heart Health Study Cohort. Sleep. 2016;39:1353-9.

37. Javaheri S, Sharma RK, Bluemke DA, Redline S. Association between central sleep apnea and left ventricular structure: the Multi-Ethnic Study of Atherosclerosis. J. Sleep Res. 2017;26(4):477-80. https://doi.org/1 $0.1111 /$ jsr.12501.

38. Wang C, Zhan X, Liang L, Abecasis GR, Lin X. Improved ancestry estimation for both genotyping and sequencing data using projection procrustes analysis and genotype imputation. Am J Hum Genet. 2015;96(6):926-37. https://doi.org/10.1016/j.ajhg.2015.04.018.

39. Sofer T, Zheng X, Gogarten SM, Laurie CA, Grinde K, Shaffer JR, et al. A fully adjusted two-stage procedure for rank-normalization in genetic association studies. Genet Epidemiol. 2019;43(3):263-75. https://doi.org/10.1002/ gepi.22188.

40. Regier AA, Farjoun Y, Larson DE, Krasheninina O, Kang HM, Howrigan DP, et al. Functional equivalence of genome sequencing analysis pipelines enables harmonized variant calling across human genetics projects. Nat Commun. 2018;9(1):4038. https://doi.org/10.1038/s41467-018-06159-4.

41. Cingolani $P$, Platts $A$, Wang $L L$, Coon M, Nguyen $T$, Wang $L$, et al. A program for annotating and predicting the effects of single nucleotide polymorphisms, SnpEff: SNPs in the genome of Drosophila melanogaster strain w1118; iso-2; iso-3. Fly (Austin). 2012;6(2):80-92. https://doi.org/10.41 61/fly.19695.
42. Kang HM, Sul JH, Service SK, Zaitlen NA, Kong S-Y, Freimer NB, et al. Variance component model to account for sample structure in genomewide association studies. Nat Genet. 2010;42:348-54.

43. Hunt SE, McLaren W, Gil L, Thormann A, Schuilenburg H, Sheppard D, et al. Ensembl variation resources. Database J Biol Databases Curation. 2018;2018: bay119. https://pubmed.ncbi.nlm.nih.gov/30576484/.

44. Dreos R, Ambrosini G, Groux R, Cavin Périer R, Bucher P. The eukaryotic promoter database in its 30th year: focus on non-vertebrate organisms. Nucleic Acids Res. 2017;45(D1):D51-5. https://doi.org/10.1093/nar/gkw1069.

45. Yevshin I, Sharipov R, Kolmykov S, Kondrakhin Y, Kolpakov F. GTRD: a database on gene transcription regulation-2019 update. Nucleic Acids Res. 2018;47(D1): D100-5. https:/pubmed.ncbi.nlm.nih.gov/30445619/.

46. Rogers MF, Shihab HA, Mort M, Cooper DN, Gaunt TR, Campbell C. FATH MM-XF: accurate prediction of pathogenic point mutations via extended features. Bioinforma Oxf Engl. 2018;34(3):511-3. https://doi.org/10.1093/ bioinformatics/bt×536.

47. di lulio J, Bartha I, Wong EHM, Yu H-C, Lavrenko V, Yang D, et al. The human noncoding genome defined by genetic diversity. Nat Genet. 2018; 50(3):333-7. https://doi.org/10.1038/s41588-018-0062-7.

48. Sundaram L, Gao H, Padigepati SR, McRae JF, Li Y, Kosmicki JA, et al. Predicting the clinical impact of human mutation with deep neural networks. Nat Genet. 2018;50(8):1161-70. https://doi.org/10.1038/s41588-01 8-0167-z.

49. Havrilla JM, Pedersen BS, Layer RM, Quinlan AR. A map of constrained coding regions in the human genome. Nat Genet. 2019;51:88-95.

50. Dozmorov MG. Epigenomic annotation-based interpretation of genomic data: from enrichment analysis to machine learning. Bioinforma Oxf Engl. 2017;33(20):3323-30. https://doi.org/10.1093/bioinformatics/btx414.

51. Chèneby J, Gheorghe M, Artufel M, Mathelier A, Ballester B. ReMap 2018: an updated atlas of regulatory regions from an integrative analysis of DNAbinding ChIP-seq experiments. Nucleic Acids Res. 2018;46(D1):D267-75. https://doi.org/10.1093/nar/gkx1092.

52. Willer CJ, Li Y, Abecasis GR. METAL: fast and efficient meta-analysis of genomewide association scans. Bioinformatics. 2010;26(17):2190-1. https:// doi.org/10.1093/bioinformatics/btq340.

53. Barbeira, A., Dickinson, S.P., Torres, J.M., Bonazzola, R., Zheng, J., Torstenson, E.S., Wheeler, H.E., Shah, K.P., Edwards, T., Garcia, T., et al. (2017). Integrating tissue specific mechanisms into GWAS summary results.

54. Zhu S, Qian T, Hoshida Y, Shen Y, Yu J, Hao K. GIGSEA: genotype imputed gene set enrichment analysis using GWAS summary level data. Bioinforma Oxf Engl. 2018.

55. GTEx Consortium, Laboratory, Data Analysis \&Coordinating Center (LDAC C)_-Analysis Working Group, Statistical Methods groups_-Analysis Working Group, Enhancing GTEx (eGTEx) groups, NIH Common Fund, NIH/NCl, NIH/ $\mathrm{NHGRI}, \mathrm{NIH} / \mathrm{NIMH}, \mathrm{NIH} / \mathrm{NIDA}$, Biospecimen Collection Source Site-NDRI, et al. Genetic effects on gene expression across human tissues. Nature. 2017:550(7675):204-13. https://doi.org/10.1038/nature24277.

56. Roadmap Epigenomics Consortium, Kundaje A, Meuleman W, Ernst J, Bilenky $M$, Yen $A$, et al. Integrative analysis of 111 reference human epigenomes. Nature. 2015;518:317-30.

57. Martens JHA, Stunnenberg HG. BLUEPRINT: mapping human blood cell epigenomes. Haematologica. 2013;98(10):1487-9. https://doi.org/10.3324/ha ematol.2013.094243.

58. Vermunt MW, Reinink P, Korving J, de Bruijn E, Creyghton PM, Basak O, et al. Large-scale identification of coregulated enhancer networks in the adult human brain. Cell Rep. 2014;9(2):767-79. https://doi.org/10.1016/j.celrep.2 014.09.023.

59. Cotto KC, Wagner AH, Feng Y-Y, Kiwala S, Coffman AC, Spies G, et al. DGldb 3.0: a redesign and expansion of the drug-gene interaction database. Nucleic Acids Res. 2018;46(D1):D1068-73. https:/doi.org/10.1093/nar/ gkx1143.

60. Stelzer G, Rosen N, Plaschkes I, Zimmerman S, Twik M, Fishilevich S, et al. The GeneCards Suite: from gene data mining to disease genome sequence analyses. Curr Protoc Bioinforma. 2016;54:1.30.1-1.30.33.

61. Corte TJ, Wort SJ, Talbot S, Macdonald PM, Hansel DM, Polkey M, et al. Elevated nocturnal desaturation index predicts mortality in interstitial lung disease. Sarcoidosis Vasc Diffuse Lung Dis. 2012;29:41-50.

62. Mohsenin V. Effects of gender on upper airway collapsibility and severity of obstructive sleep apnea. Sleep Med. 2003;4(6):523-9. https://doi.org/10.101 6/S1389-9457(03)00168-0. 
63. López-Doménech G, Serrat R, Mirra S, D’Aniello S, Somorjai I, Abad A, et al The Eutherian Armcx genes regulate mitochondrial trafficking in neurons and interact with Miro and Trak2. Nat Commun. 2012;3(1):814. https://doi. org/10.1038/ncomms1829.

64. Serrat R, López-Doménech G, Mirra S, Quevedo M, Garcia-Fernàndez J, Ulloa $F$, et al. The non-canonical Wnt/PKC pathway regulates mitochondrial dynamics through degradation of the arm-like domain-containing protein Alex3. PLoS One. 2013;8(7):e67773. https://doi.org/10.1371/journal.pone. 0067773.

65. Cartoni R, Norsworthy MW, Bei F, Wang C, Li S, Zhang Y, et al. The mammalian-specific protein Armcx1 regulates mitochondrial transport during axon regeneration. Neuron. 2016;92:1294-307.

66. Yoon JC, Ng A, Kim BH, Bianco A, Xavier RJ, Elledge SJ. Wnt signaling regulates mitochondrial physiology and insulin sensitivity. Genes Dev. 2010; 24(14):1507-18. https://doi.org/10.1101/gad.1924910.

67. Ramezani RJ, Stacpoole PW. Sleep disorders associated with primary mitochondrial diseases. J Clin Sleep Med JCSM Off Publ Am Acad Sleep Med. 2014;10:1233-9.

68. Waypa GB, Smith KA, Schumacker PT. O2 sensing, mitochondria and ROS signaling: the fog is lifting. Mol Aspects Med. 2016;47-48:76-89.

69. Pialoux V, Hanly PJ, Foster GE, Brugniaux JV, Beaudin AE, Hartmann SE, et al. Effects of exposure to intermittent hypoxia on oxidative stress and acute hypoxic ventilatory response in humans. Am J Respir Crit Care Med. 2009; 180(10):1002-9. https://doi.org/10.1164/rccm.200905-06710C.

70. MacFarlane PM, Mitchell GS. Respiratory long-term facilitation following intermittent hypoxia requires reactive oxygen species formation. Neuroscience. 2008;152(1):189-97. https://doi.org/10.1016/j.neuroscience.2 007.12.003.

71. Kim SR, Kim DI, Kim SH, Lee H, Lee KS, Cho SH, et al. NLRP3 inflammasome activation by mitochondrial ROS in bronchial epithelial cells is required for allergic inflammation. Cell Death Dis. 2014;5(10):e1498. https://doi.org/10.103 8/cddis.2014.460.

72. Ichimura H, Parthasarathi K, Quadri S, Issekutz AC, Bhattacharya J. Mechanooxidative coupling by mitochondria induces proinflammatory responses in lung venular capillaries. J Clin Invest. 2003;111(5):691-9. https://doi.org/10.11 72/JCI17271.

73. Kim JS, Podolanczuk AJ, Borker P, Kawut SM, Raghu G, Kaufman JD, et al. Obstructive sleep apnea and subclinical interstitial lung disease in the MultiEthnic Study of Atherosclerosis (MESA). Ann Am Thorac Soc. 2017;14(12): 1786-95. https://doi.org/10.1513/AnnalsATS.201701-0910C.

74. Gopisetty G, Thangarajan R. Mammalian mitochondrial ribosomal small subunit (MRPS) genes: a putative role in human disease. Gene. 2016;589(1): 27-35. https://doi.org/10.1016/j.gene.2016.05.008.

75. Bousquet PA, Sandvik JA, Arntzen M $\varnothing$, Jeppesen Edin NF, Christoffersen S, Krengel $U$, et al. Hypoxia strongly affects mitochondrial ribosomal proteins and translocases, as shown by quantitative proteomics of HeLa cells. Int J Proteomics. 2015;2015:678527.

76. Seo M, Qiu W, Bailey W, Criner GJ, Dransfield MT, Fuhlbrigge AL, et al. Genomics and response to long-term oxygen therapy in chronic obstructive pulmonary disease. J Mol Med Berl Ger. 2018;96:1375-85.

77. Baik I, Seo HS, Yoon D, Kim SH, Shin C. Associations of sleep apnea, NRG1 polymorphisms, alcohol consumption, and cerebral white matter hyperintensities: analysis with genome-wide association data. Sleep. 2015; 38(7):1137-43. https://doi.org/10.5665/sleep.4830.

78. Finigan JH, Mishra R, Vasu VT, Silveira LJ, Nethery DE, Standiford TJ, et al. Bronchoalveolar lavage neuregulin-1 is elevated in acute lung injury and correlates with inflammation. Eur Respir J. 2013;41(2):396-401. https://doi. org/10.1183/09031936.00004912.

79. Liu J, Nethery D, Kern JA. Neuregulin-1 induces branching morphogenesis in the developing lung through a P13K signal pathway. Exp Lung Res. 2004; 30:465-78.

80. Venugopal R, Galam L, Cox R, Fukumoto J, Cho Y, Parthasarathy PT, et al. Inflammasome inhibition suppresses alveolar cell permeability through retention of neuregulin-1 (NRG-1). Cell Physiol Biochem Int J Exp Cell Physiol Biochem Pharmacol. 2015;36(5):2012-24. https://doi.org/10.1159/ 000430169.

81. Paatero I, Jokilammi A, Heikkinen PT, Iljin K, Kallioniemi O-P, Jones FE, et al. Interaction with ErbB4 promotes hypoxia-inducible factor-1a signaling. J Biol Chem. 2012;287:9659-71.

82. Kyotani Y, Ota H, Itaya-Hironaka A, Yamauchi A, Sakuramoto-Tsuchida S, Zhao J, et al. Intermittent hypoxia induces the proliferation of rat vascular smooth muscle cell with the increases in epidermal growth factor family and erbB2 receptor. Exp Cell Res. 2013;319:3042-50.

83. Gamazon ER, Konkashbaev A, Derks EM, Cox NJ, Lee Y. Evidence of selection on splicing-associated loci in human populations and relevance to disease loci mapping. Sci Rep. 2017;7(1):5980. https://doi.org/10.1038/s41 598-017-05744-9.

84. Stauffer TP, Guerini D, Carafoli E. Tissue distribution of the four gene products of the plasma membrane Ca2+ pump. A study using specific antibodies. J Biol Chem. 1995;270(20):12184-90. https://doi.org/10.1074/jbc.2 70.20.12184.

85. Schuh K, Quaschning T, Knauer S, Hu K, Kocak S, Roethlein N, et al. Regulation of vascular tone in animals overexpressing the sarcolemmal calcium pump. J Biol Chem. 2003;278(42):41246-52. https://doi.org/10.1074/ jbc.M307606200.

86. Kell AM, Gale M. RIG-I in RNA virus recognition. Virology. 2015;479-480:110-21.

87. Gharib SA, Hayes AL, Rosen MJ, Patel SR. A pathway-based analysis on the effects of obstructive sleep apnea in modulating visceral fat transcriptome. Sleep. 2013;36(1):23-30. https://doi.org/10.5665/sleep.2294.

88. Herriges JC, Verheyden JM, Zhang Z, Sui P, Zhang Y, Anderson MJ, et al. FGF-regulated ETV transcription factors control FGF-SHH feedback loop in lung branching. Dev Cell. 2015;35(3):322-32. https://doi.org/10.1016/j. devcel.2015.10.006.

89. Wollenick K, Hu J, Kristiansen G, Schraml P, Rehrauer H, BerchnerPfannschmidt $U$, et al. Synthetic transactivation screening reveals ETV4 as broad coactivator of hypoxia-inducible factor signaling. Nucleic Acids Res. 2012;40(5):1928-43. https://doi.org/10.1093/nar/gkr978.

90. Shu W, Yang H, Zhang L, Lu MM, Morrisey EE. Characterization of a new subfamily of winged-helix/forkhead (Fox) genes that are expressed in the lung and act as transcriptional repressors. J Biol Chem. 2001;276(29):2748897. https://doi.org/10.1074/jbc.M100636200.

91. Chokas AL, Trivedi CM, Lu MM, Tucker PW, Li S, Epstein JA, et al. Foxp1/2/4NuRD interactions regulate gene expression and epithelial injury response in the lung via regulation of interleukin-6. J Biol Chem. 2010;285(17):1330413. https://doi.org/10.1074/jbc.M109.088468.

92. Xu S, Liu P, Chen Y, Chen Y, Zhang W, Zhao H, et al. Foxp2 regulates anatomical features that may be relevant for vocal behaviors and bipedal locomotion. Proc Natl Acad Sci U S A. 2018;115(35):8799-804. https://doi. org/10.1073/pnas.1721820115.

93. Stanić D, Dhingra RR, Dutschmann M. Expression of the transcription factor FOXP2 in brainstem respiratory circuits of adult rat is restricted to upperairway pre-motor areas. Respir Physiol Neurobiol. 2018;250:14-8. https://doi. org/10.1016/j.resp.2018.01.014.

94. Burgold T, Voituron N, Caganova M, Tripathi PP, Menuet C, Tusi BK, et al. The H3K27 demethylase JMJD3 is required for maintenance of the embryonic respiratory neuronal network, neonatal breathing, and survival. Cell Rep. 2012;2(5):1244-58. https://doi.org/10.1016/j.celrep.2012.09.013.

95. Smith JC, Ellenberger HH, Ballanyi K, Richter DW, Feldman JL. Pre-Bötzinger complex: a brainstem region that may generate respiratory rhythm in mammals. Science. 1991;254(5032):726-9. https://doi.org/10.1126/science.1683005.

96. Chen Y, Lin M, Su M, Chin C, Huang K, Chang Y. Global histone H3K23/ H3K36 hypoacetylation and HDAC1 up-regulation are associated with disease severity and adverse consequences in obstructive sleep apnea patients. Am J Respir Crit Care Med. 2018;197:A6411.

97. Satoh T, Takeuchi O, Vandenbon A, Yasuda K, Tanaka Y, Kumagai Y, et al. The Jmjd3-Irf4 axis regulates M2 macrophage polarization and host responses against helminth infection. Nat Immunol. 2010;11(10):936-44. https://doi.org/10.1038/ni.1920.

98. De Santa F, Totaro MG, Prosperini E, Notarbartolo S, Testa G, Natoli G. The histone $\mathrm{H} 3$ lysine-27 demethylase Jmjd3 links inflammation to inhibition of polycomb-mediated gene silencing. Cell. 2007;130(6):1083-94. https://doi. org/10.1016/j.cell.2007.08.019.

99. Li Q, Wang HY, Chepelev I, Zhu Q, Wei G, Zhao K, et al. Stage-dependent and locus-specific role of histone demethylase Jumonji D3 (JMJD3) in the embryonic stages of lung development. PLoS Genet. 2014;10(7):e1004524. https://doi.org/10.1371/journal.pgen.1004524.

100. Shalaby NA, Pinzon JH, Narayanan AS, Jin EJ, Ritz MP, Dove RJ, et al. JmjC domain proteins modulate circadian behaviors and sleep in Drosophila. Sci Rep. 2018;8(1):815. https://doi.org/10.1038/s41598-017-18989-1.

101. Shmakova A, Batie M, Druker J, Rocha S. Chromatin and oxygen sensing in the context of JmjC histone demethylases. Biochem. J. 2014;462(3):385-95. https://doi.org/10.1042/BJ20140754. 
102. Hancock RL, Masson N, Dunne K, Flashman E, Kawamura A. The activity of JmjC histone lysine demethylase KDM4A is highly sensitive to oxygen concentrations. ACS Chem. Biol. 2017;12(4):1011-9. https://doi.org/10.1021/a cschembio.6b00958.

103. Yan SF, Zou YS, Gao Y, Zhai C, Mackman N, Lee SL, et al. Tissue factor transcription driven by Egr-1 is a critical mechanism of murine pulmonary fibrin deposition in hypoxia. Proc Natl Acad Sci U S A. 1998;95:8298-303.

104. Thomason HA, Dixon MJ, Dixon J. Facial clefting in Tp63 deficient mice results from altered Bmp4, Fgf8 and Shh signaling. Dev Biol. 2008;321(1): 273-82. https://doi.org/10.1016/j.ydbio.2008.06.030.

105. Robison JG, Otteson TD. Increased prevalence of obstructive sleep apnea in patients with cleft palate. Arch. Otolaryngol. Head Neck Surg. 2011;137(3): 269-74. https://doi.org/10.1001/archoto.2011.8.

106. Obeidat M, Miller S, Probert K, Billington CK, Henry AP, Hodge E, et al. GSTC $D$ and INTS12 regulation and expression in the human lung. PLoS One. 2013;8(9):e74630. https://doi.org/10.1371/journal.pone.0074630.

\section{Publisher's Note}

Springer Nature remains neutral with regard to jurisdictional claims in published maps and institutional affiliations.

Ready to submit your research? Choose BMC and benefit from:

- fast, convenient online submission

- thorough peer review by experienced researchers in your field

- rapid publication on acceptance

- support for research data, including large and complex data types

- gold Open Access which fosters wider collaboration and increased citations

- maximum visibility for your research: over $100 \mathrm{M}$ website views per year

At $\mathrm{BMC}$, research is always in progress.

Learn more biomedcentral.com/submissions 